\title{
Does advance contact with research participants increase response to questionnaires: an updated systematic review and meta-analysis
}

Benjamin Woolf ${ }^{1,2,3^{*}}$ and Phil Edwards ${ }^{3}$

\begin{abstract}
Background: Questionnaires remain one of the most common forms of data collection in epidemiology, psychology and other human-sciences. However, results can be badly affected by non-response. One way to potentially reduce non-response is by sending potential study participants advance communication. The last systematic review to examine the effect of questionnaire pre-notification on response is 10 years old, and lacked a risk of bias assessment.

Objectives: Update the section of the Cochrane systematic review, Edwards et al. (2009), on pre-notification to include 1) recently published studies, 2) an assessment of risk of bias, 3) Explore if heterogeneity is reduced by: delay between pre-contact and questionnaire delivery, the method of pre-contact, if pre-contact and questionnaire delivery differ, if the pre-contact includes a foot-in-the-door manipulation, and study's the risk of bias.

Methods: Inclusion criteria: population: any population, intervention: comparison of some type of pre-notification, comparison group: no pre-notification, outcome: response rates. Study design: randomised controlled trails. Exclusion criteria: NA. Data sources: Studies which cited or were included in Edwards et al. (2009); We additionally searched: CINAHL, Web of Science, PsycInfo, MEDLINE, EconLit, EMBASE, Cochrane Central, Cochrane CMR, ERIC, and Sociological Abstracts. The searches were implemented in June 2018 and May 2021. Study screening: a single reviewer screened studies, with a random $10 \%$ sample independently screened to ascertain accuracy. Data extraction: data was extracted by a single reviewer twice, with a week between each extraction. Risk of Bias: within studies bias was assessed using the Cochrane Risk of Bias tool (ROB1) by a single unblinded reviewer, across studies bias was assessed using funnel plots. Synthesis Method: study results were meta-analysed with a random effects model using the final response rate as the outcome. Evaluation of Uncertainty: Uncertainty was evaluated using the GRADE approach.

Results: One hundred seven trials were included with 211,802 participants. Over-all pre-notification increased response, $\mathrm{OR}=1.33$ (95\% Cl: $1.20-1.47)$. However, there was a large amount of heterogeneity $\left(1^{2}=97.1 \%\right)$, which was not explained by the subgroup analyses. In addition, when studies at high or unclear risk of bias were excluded the effect was to reduced $\mathrm{OR}=1.09$ (95\% Cl: 0.99-1.20). Because of the large amount of heterogeneity, even after restricting to low risk of bias studies, there is still moderate uncertainty in these results.
\end{abstract}

\footnotetext{
${ }^{*}$ Correspondence: Benjamin.woolf@bristol.ac.uk

${ }^{1}$ Department of Psychological Science, University of Bristol, 5 Priory Road,

Bristol, UK

Full list of author information is available at the end of the article
}

(C) The Author(s) 2021. Open Access This article is licensed under a Creative Commons Attribution 4.0 International License, which permits use, sharing, adaptation, distribution and reproduction in any medium or format, as long as you give appropriate credit to the original author(s) and the source, provide a link to the Creative Commons licence, and indicate if changes were made. The images or other third party material in this article are included in the article's Creative Commons licence, unless indicated otherwise in a credit line to the material. If material is not included in the article's Creative Commons licence and your intended use is not permitted by statutory regulation or exceeds the permitted use, you will need to obtain permission directly from the copyright holder. To view a copy of this licence, visit http://creativecommons.org/licenses/by/4.0/. The Creative Commons Public Domain Dedication waiver (http://creativeco mmons.org/publicdomain/zero/1.0/) applies to the data made available in this article, unless otherwise stated in a credit line to the data. 
Conclusions: Using the GRADE evaluation, this review finds moderate evidence that pre-notification may not have an effect on response rates.

Funding: Economic and Social Research Council.

Preregistration: None.

Keywords: Pre-notification, Systematic review, Questionnaire response

\section{Introduction}

Questionnaires have been one of the most common methods of data collection across the social and medical sciences. For example, in epidemiology pen and paper questionnaires alone were used in $29.2 \%$ of over 2000 analytic epidemiological studies included in a review of articles published in high-impact medical journals between 2008 and 2009 [1]. Likewise, about a third of empirical research published in management and accounting journals use questionnaires, and a review of a top social psychology journal found that over $91 \%$ of empirical studies published in the second half of 2017 used some form of questionnaire [2,3].

Inherent in using questionnaires is a risk of nonresponse. Potential participants, for example, might forget to complete questionnaires, and research ethics requires a right to refuse participation. Non-response can negatively impact on studies in three major ways: Firstly, non-response can introduce selection bias [4]. Secondly, even in the absence of selection bias, because nonresponse reduces the number of participants recruited into a study, non-response increases risk of random error (i.e. reduces statistical power and precision). Finally, nonresponse increases study costs [5].

It is therefore important to minimise non-response. One potential method is for the study team to contact potential participants in advance of them receiving the questionnaire (questionnaire pre-notification). In 2009, Edwards et al. published the third update of a 2003 Cochrane systematic review of randomised control trials evaluating methods of reducing non-response in both postal and electronic questionnaires [6]. They found that pre-contact increased response when compared to no pre-contact $(\mathrm{OR}=1.5,95 \%$ CI $1.26-1.78$, for response after first questionnaire administration, and $\mathrm{OR}=1.45$, 95\% CI 1.29-1.63 for response after final questionnaire administration). However, Edwards et al. (2009) did not assess the risk of bias in or across the included studies, and is now 10 years old, so therefore does not include research published in the last decade. In addition, there was substantial heterogeneity among the study results $\left(p<0.000001 ; \mathrm{I}^{2}=91 \%\right.$ for the response after the first questionnaire administration, and $p<0.00001$; $\mathrm{I}^{2}=89 \%$ for the response after the final questionnaire administration).
There is therefore a need for an updated review which includes recently published studies, an assessment of bias risk in and across included studies. This review will:

1. Update Edwards et al. (2009)'s systematic review and meta-analysis of randomised control trials examining the effect on non-response of pre-notification relative to no pre-notification (in any population) so that it includes papers published in the last decade.

2. To carry out an assessment of the risk of bias (i) in and (ii) across included studies.

3. To examine the extent to which between study heterogeneity is explained by: (A) the delay between pre-contact and questionnaire delivery, (B) method of pre-contact, $(C)$ if pre-contact differs from questionnaire delivery, (D) if the pre-contact includes a foot-in-the-door manipulation (required participants to do something to receive the questionnaire), and (E) differences in the risk of bias of included studies, through conducting a subgroup analysis.

\section{Methods}

\section{Protocol and registration}

The methodology of the review and analysis was approved in advance by the LSHTM epidemiology MSc course directors. A copy of this form, approved on 21/03/2018, can be found in Supplementary Table 1. However the study was not otherwise registered.

This study received ethics approval from the London School of Hygiene and Tropical Medicine MSc Research Ethics Committee on 26/03/2018. This study has been written in accordance with PRISMA-2020 [7].

\section{Eligibility criteria \\ Inclusion criteria}

Types of population: This study followed Edwards et al. (2009) in using data from "[a]ny population (e.g. patients or healthcare providers and including any participants of non-health studies)." This should maximise generalisability over different contexts.

Types of interventions: interventions must include some type of questionnaire pre-contact (pre-notification, advance letter/email/text/phone call or other 
co-referring term). No restriction is placed on the type of questionnaire pre-notification.

Comparison group: Included studies need to be able to make a direct comparison of the effect of questionnaire pre-notification vs no pre-notification (i.e. include at least one arm which received identical treatment to the pre-notification arm other than not receiving the pre-notification).

Types of outcome measures: The proportion or number of completed, or partially completed questionnaires returned after all follow-up contacts were complete.

Types of study design: Any randomised control trial evaluating a method of advanced contact to increase response to questionnaires. The inclusion of only randomised control trials should on average eliminate risk of confounding biasing estimates within studies.

\section{Exclusion criteria}

There are no exclusion criteria.

\section{Information sources}

Relevant studies identified by Edwards et al. (2009). A detailed description of the information sources, e.g. databases with dates of coverage, used in this study are in its methods section and Supplementary Tables, which can be freely accessed in the Cochrane Library (https://www. cochranelibrary.com/cdsr/doi/10.1002/14651858.MR000 008.pub4/full).In addition, the references of all included studies, and any citation they, or Edwards et al. (2009), had received by the $28 / 6 / 2018$ were checked for meeting the eligibility criteria.

The search strategy was developed by modifying the strategy used by Edwards et al. (2009), to make it more sensitive and specific to detecting studies examining questionnaire pre-notification, by adding terms denoting types of pre-notification, and removing terms relating to other methods. The strategy was validated by inputting the new terms into Google Scholar, and checking that it detected all relevant studies included in Edwards et al. (2009). The specific search terms are presenting in Supplementary Table 2 . The search strategy was implemented in the same data-bases used in Edwards et al. (2009) from the date they were last searched till the present day. Specifically, the following databases were searched (with date restrictions in brackets): CINAHL (2007.12-2018.6); Dissertation \& Thesis, Social Science Citation Index, Science Citation Index, and Index to Scientific \& Technical Proceedings in Web of Science (2008.1-2018.6); PsycInfo (2008.1-2018.6); MEDLINE (2007.1-2018.6); EconLit (2008.1-2018.6); EMBASE (2008.1-2018.6);
Cochrane Central (2008.1-2018.6); Cochrane CMR (2008.1-2018.6); ERIC (2008.1-2018.6); and Sociological Abstracts (2007.1-2018.6). After consultation with the LSHTM library, two databases searched by Edwards et al. (2009) (National Research Register and Social Psychological Educational Criminological Trials Register) were not searched because they were both deemed inaccessible and no longer operational. Any relevant reviews found in the literature search were examined for relevant studies.

.Finally, because the search was out of date, the search terms were re-implemented in CINAHL (2018.1-2021.5); Dissertation \& Thesis, Social Science Citation Index, Science Citation Index, and Index to Scientific \& Technical Proceedings in Web of Science (2018.1-2021.5); PsycInfo (2018.1-2021.5); MEDLINE (2018.1-2021.5); EMBASE (2018.1-2021.5). The search was not re-run in Cochrane Central, Cochrane CMR, ERIC, Sociological Abstracts, or EconLit because they accounted for only $2.5 \%$ of studies identified in a database in 2018.

Non-English papers were translated using Google Translate.

\section{Study selection}

The eligibility assessment was conducted by one reviewer following a standardised procedure. This process was repeated on a random $10 \%$ by a second reviewer with 99.7\% agreement. Citations were uploaded onto Covidence (http://www.covidence.org/), a website specially designed for paper screening by the Cochrane Collaboration. Covidence automatically identified duplicates of citation/abstracts, which were then manually checked for errors.

Studies were first screened based on abstracts and titles, then full text. This process was repeated for any study which was referenced by or itself cited by an included study, and on the content of any potentially relevant review identified in the search.

\section{Data collection process}

A standardised data extraction sheet (Supplementary Table 3) was developed. The sheet was pilot tested on 10 randomly chosen studies from Edwards et al. (2009). One reviewer extracted data from included studies. To minimise transcription errors, this process was duplicated by the same reviewer 1 week later. Disagreements were resolved by extracting information for a third time and using the third extraction as the definitive extraction.

To check for duplication studies which shared at least one author were compared based on similarity of study population, date, and methodology. Duplicate trials were treated as a single study in the meta-analysis. 


\section{Data items}

Information extracted for each included trial comprised 5 domains:

1) Information on the inclusion criteria: The study design, nature of the control arm, information on the intervention arm(s), information about the outcome measurement (the number of responses, and/or the response rate, in each arm).

2) Information on risk of bias: how the allocation sequence was generated, information of allocation concealment, blinding of participants and personnel, blinding of outcome assessors, any incomplete outcome data, information on other possible sources of bias (e.g. source of funding).

3) Information on the participants: the total number of participants, numbers in each arm, setting, country.

4) Information on the outcome: number of items returned, or response rate, in each arm.

5) Other information: the time from the sending of prenotification to questionnaire, if it includes a foot-inthe-door manipulation, the type of questionnaire administration, the type of pre-contact.

\section{Risk of bias in individual studies}

Assessment of risk of bias within each study was conducted by one unblinded reviewer. Information on risk of bias was extracted twice with a one-week gap between each extraction, and conflicts were handled by using the results of a third extraction. Authors included in the 2018 search were contacted for extra information about study bias risk, and still existent copies of communication from Edwards et al. (2009) were examined.

Bias was evaluated using the Cochrane Risk of Bias tool [8]. The tool involves rating the risk of bias across 7 domains (random sequence generation, allocation concealment, blinding of participant and personnel, blinding of outcome assessment, incomplete outcome data, selective reporting, and other biases) at the outcome level. Within each domain, the studies were ranked as either high or low risk of bias, depending on the description of the study provided. If insufficient information was provided to form a decision, studies were designated as 'unclear' risk of bias. Studies were classified as at a low risk of bias if they had a low risk in all domains, at a high risk of bias if at a high risk in one domain, and were otherwise classified as having an unclear risk of bias. A full description of the tool can be found in chapter 8 of the Cochrane Handbook [8]. Results are stratified based on Risk of Bias score.
Summary measures, and planned methods of results synthesis

The primary summary measure of association estimated was the ratio of the odds (OR) of response in the treatment groups compared with the odds of response in the control group.

In line with Edwards et al. (2009), the meta-analyses were performed by comparing the ORs using a randomeffects model. The analysis was performed on an intention-to-treat basis. Outcomes were only included if they occurred within the period of follow up.

The results were synthesised in a meta-analysis conducted using STATA 15, using the 'metan' command [9]. To be consistent with Edwards et al. (2009), a random effects meta-analysis was used. Heterogeneity was assessed using the Cochran-Q Chi [2] statistical test for heterogeneity, and the $\mathrm{I}^{2}$ statistic [10]. Results were presented using a forest plot.

To test the hypothesis that heterogeneity is explained by 1 ) the length of time between pre-contact and questionnaire, 2) method of pre-contact, 3) if pre-contact and questionnaire delivery differ, 4) if the pre-contact includes a foot-in-the-door manipulation, four planned subgroup analyses were conducted by separately stratifying the meta-analysis on these factors. Studies in which participants were not all assigned to the same type of pre-notification were excluded.

\section{Risk of bias across studies}

Risk of bias across studies was assessed with funnel plots. Asymmetry was investigated informally, by visually assessing how symmetrical the plots are around the effect estimate, and formally, using Harbord's test. Funnel plots were created using the 'metafunnel' command in STATA. Because ORs are naturally correlated with their standard error, response rates were used instead of ORs [9].

\section{Assessment of certainty in the body of evidence}

Outcome level limitations were evaluated using the GRADE approach [11] for both the overall estimate, and the estimate for studies at low risk of bias.

\section{Results \\ Study section}

A total of 103 papers, reporting a total of 107 trials, were identified for inclusion in the review. The search resulted in a total of 35,931 citations, including 14,207 duplications. Eight reviews (Supplementary Table 4) were included in the search and checked for citations. The reasons for exclusions are stated in Fig. 1 and Supplementary Table 5 . The numbers identified and excluded at each stage are described Fig. 1 . After re-reading the 


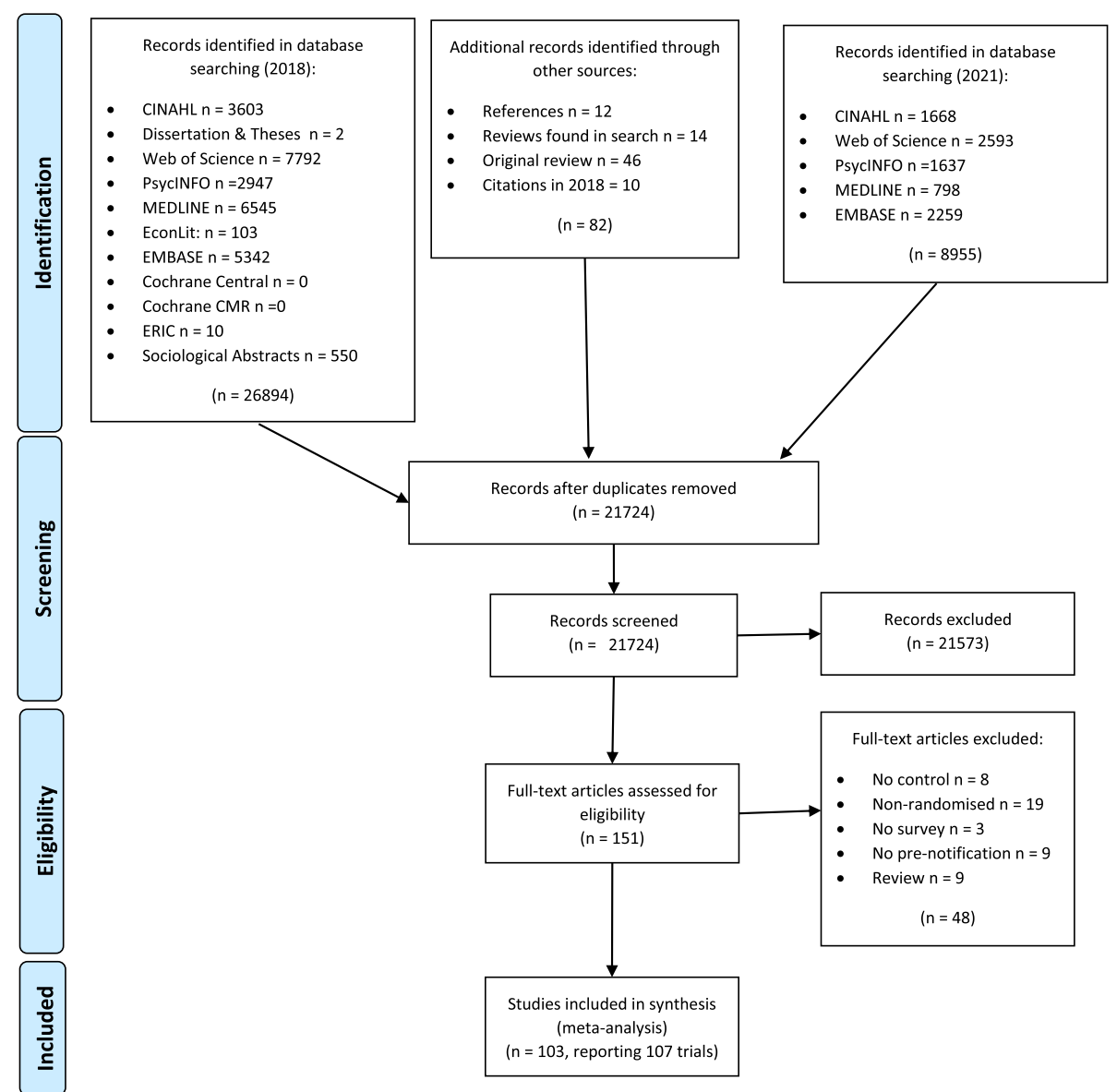

Fig. 1 Flow diagram of study inclusion

reports, and contacting study authors, five studies (Temple-Smith 1998 [12]; Waisanen 1954 [13]; Wright 1995 [14]; Wynn 1985 [15]) which were included in Edwards et al. (2009) were excluded for not having randomised participants to receive or not receive a pre-notification. No duplicates were identified during data extraction. Overall, the updated review now includes 60 more studies than Edwards et al. 2009; increasing the number of participants from 79,651 to 364,527 .

\section{Study characteristics}

Of the included studies, 32 (31.1\%) were factorial designs. 60 (58.3\%) were conducted in North America, $33(32.0 \%)$ in Europe. Two (1.9\%) were conducted in East Asia (Hong Kong and Thailand), 7 (6.7\%) in Australia, one study did not state where it was conducted, and none were conducted in South America or Africa. 37 (35.9\%) studies used samples of the general population. 13 (12.6\%) were students or alumni, 14 (13.6\%) were nested in other studies, 20 (19.4\%) used medical or academic staff, 15 (14.5\%) occupational samples, and 7 (6.7\%) samples had some type of commercial basis. Approximately a third of questionnaires were health or epidemiology related. 6 (5.8\%) trials were published prior to $1970,8(7.8 \%)$ in the 1970 's, $17(16.5 \%)$ in the 1980 's, 20 (19.4\%) in the 1990's, 22 (21.4\%) in the 2000's, $28(27.2 \%)$ in the 2010 's, and two $(1.9 \%)$ in the 2020 s. One study was not written in English.

$85(79.4 \%)$ of the pre-notifications were posted. 19 $(17.8 \%)$ of the others were telephone, with a few delivered by email $(n=7,6.5 \%)$ or text message $(n=7,6.5 \%)$. Only 17 (15.9\%) trials reported a pre-notification which included a foot-in-the-door manipulation. 28 (26.2\%) trails had a delay of less than 1 week, 33 (30.8\%) had a delay of 1 week, 11 (10.3\%) of 2 weeks. One $(0.9 \%)$ for delays of 3 weeks, 5 weeks and 6 weeks. $70(65.4 \%)$ trails administered the questionnaire by mail, 24 (22.4\%) over the phone, $12(11.2 \%)$ by email or online, and one used interviews. The characteristics of the included studies are described in detail in Table 1. 


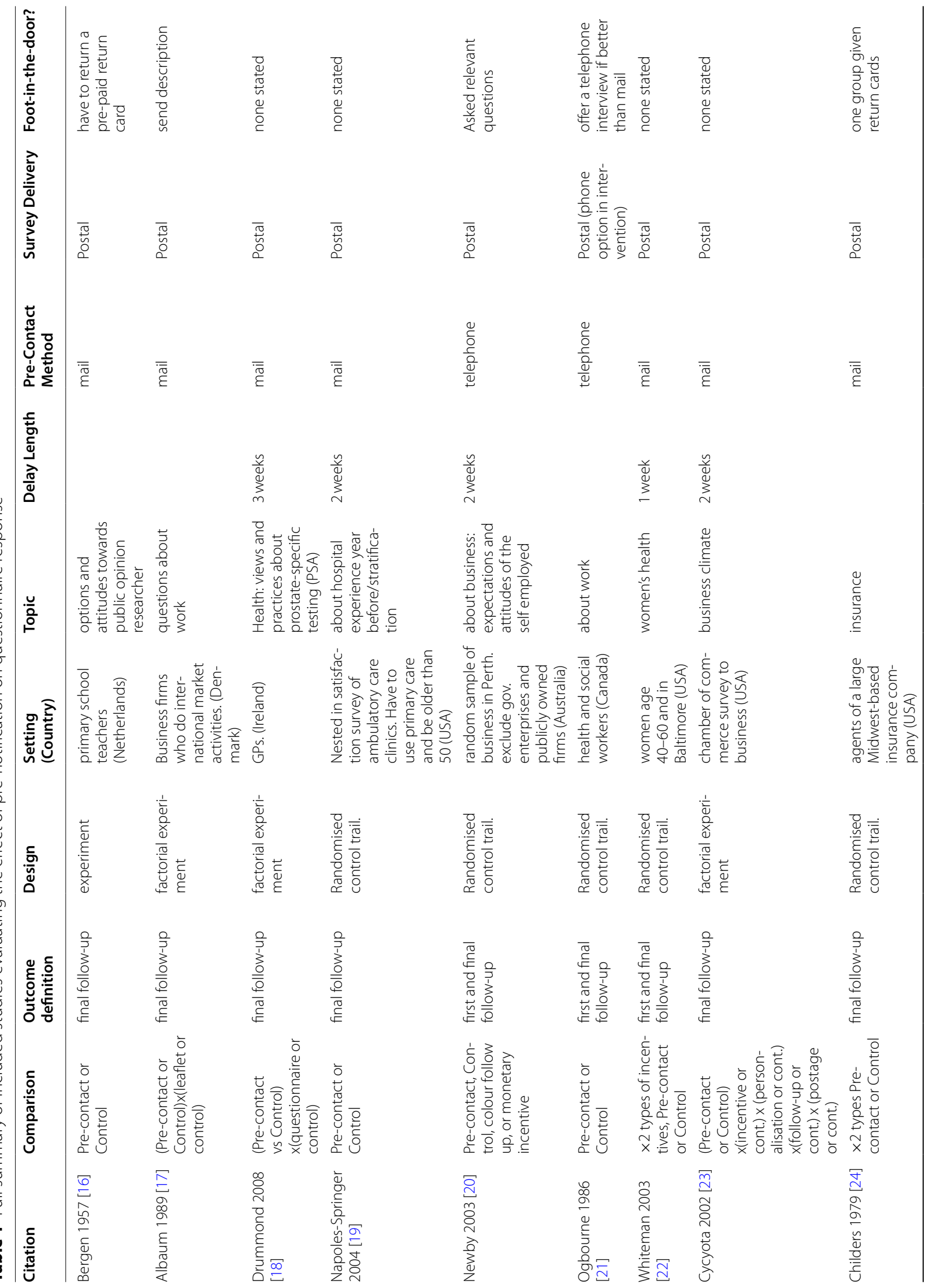




\begin{tabular}{|c|c|c|c|c|c|c|c|c|c|c|c|}
\hline 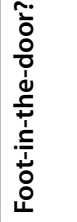 & 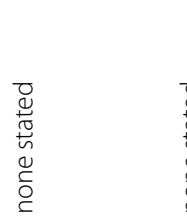 & 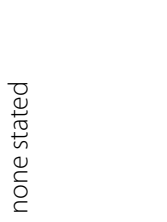 & 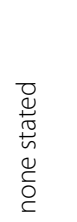 & 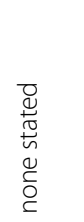 & 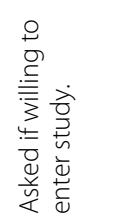 & 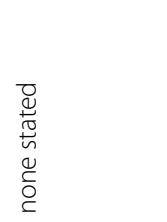 & 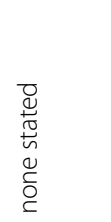 & 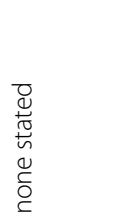 & 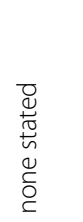 & 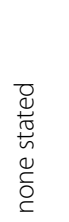 & 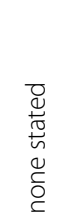 \\
\hline 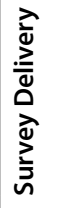 & 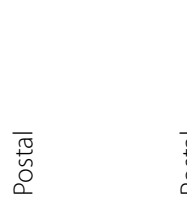 & $\begin{array}{l}\overline{\widetilde{J}} \\
\stackrel{\Delta}{\circ}\end{array}$ & 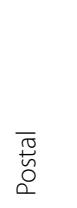 & 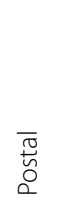 & 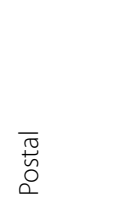 & $\begin{array}{l}\overline{\widetilde{0}} \\
\stackrel{\circ}{\circ} \\
\therefore\end{array}$ & $\begin{array}{l}\overline{\widetilde{0}} \\
\stackrel{\circ}{\circ} \\
\triangle\end{array}$ & 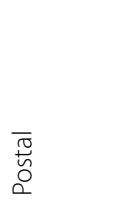 & 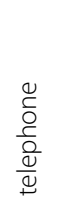 & 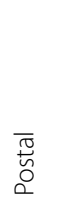 & 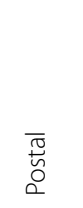 \\
\hline 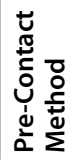 & $\overline{\overline{\bar{\sigma}}}$ & 言 & $\overline{\overline{\bar{\varepsilon}}}$ & $\stackrel{\overline{\bar{\sigma}}}{\stackrel{\varepsilon}{\varepsilon}}$ & 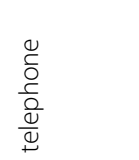 & $\stackrel{\overline{\bar{\sigma}}}{\stackrel{\varepsilon}{\varepsilon}}$ & 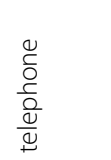 & 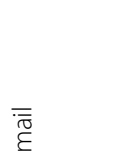 & $\stackrel{\overline{\overline{\widetilde{\sigma}}}}{\xi}$ & $\overline{\overline{\bar{\sigma}}}$ & $\overline{\overline{\tilde{\varepsilon}}}$ \\
\hline 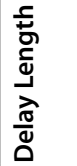 & 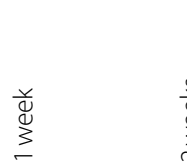 & 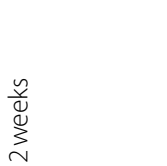 & 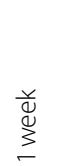 & 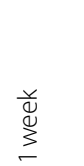 & 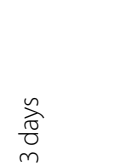 & 弟 & 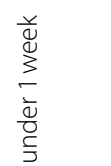 & 总 & & $\begin{array}{l}\stackrel{n}{\widehat{d}} \\
\frac{\sigma}{\sigma}\end{array}$ & $\begin{array}{l}\stackrel{\sim}{\widehat{J}} \\
\substack{0 \\
n}\end{array}$ \\
\hline 产 & 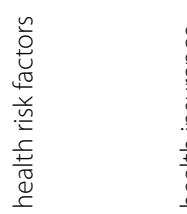 & 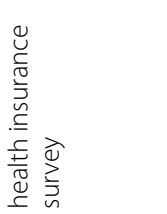 & 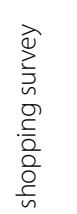 & $\begin{array}{l}\text { ओे } \\
2 \\
\bar{y} \\
0 \\
\cdot \frac{0}{0} \\
\frac{0}{0} \\
\frac{0}{n}\end{array}$ & 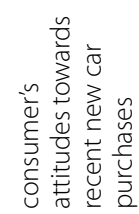 & 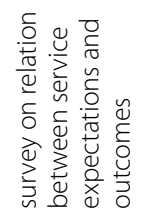 & 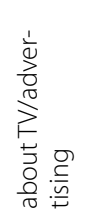 & 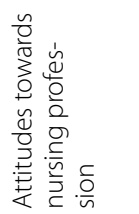 & 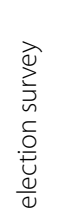 & 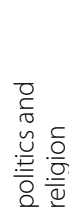 & 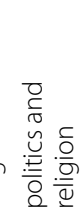 \\
\hline 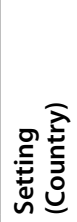 & 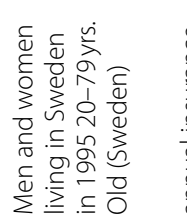 & 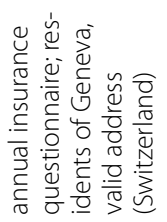 & 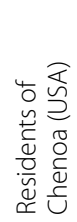 & 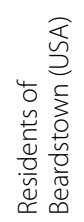 & 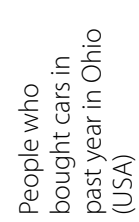 & 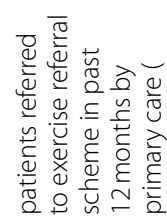 & 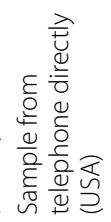 & 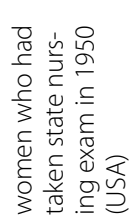 & 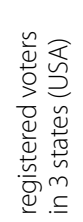 & 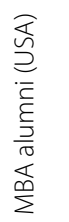 & 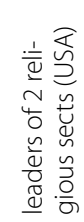 \\
\hline 点 & 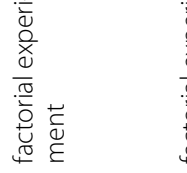 & 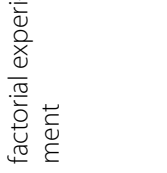 & 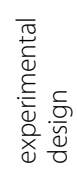 & 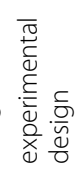 & 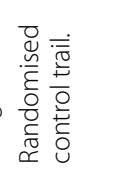 & 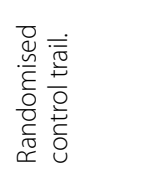 & 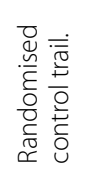 & 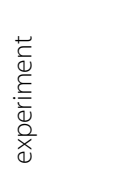 & 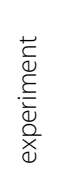 & 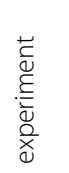 & 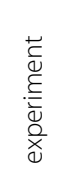 \\
\hline 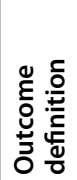 & 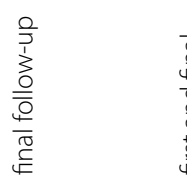 & 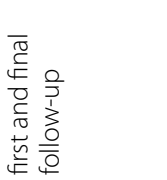 & 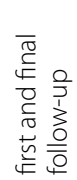 & 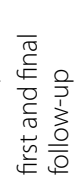 & 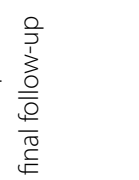 & 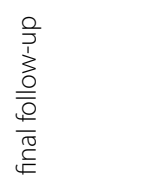 & 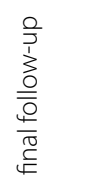 & 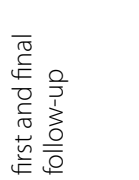 & 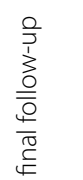 & 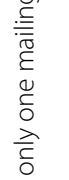 & 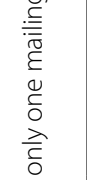 \\
\hline 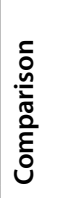 & 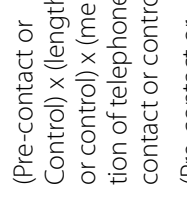 & 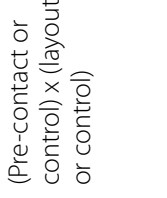 & 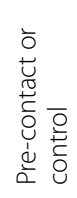 & 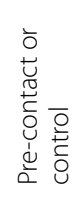 & 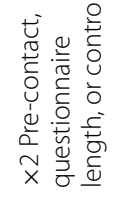 & 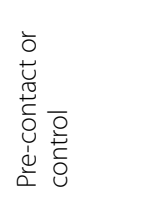 & 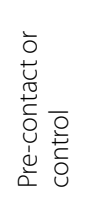 & 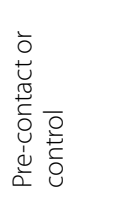 & 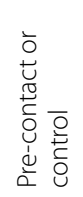 & 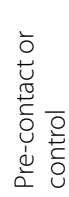 & 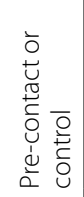 \\
\hline ס̊ & 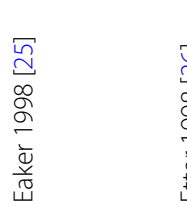 & 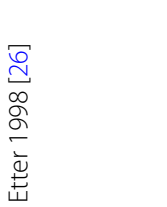 & 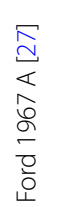 & $\begin{array}{l}\nwarrow \\
\grave{d} \\
\infty \\
o \\
o \\
\frac{0}{0} \\
\frac{0}{0}\end{array}$ & 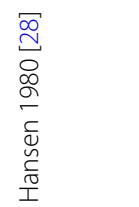 & 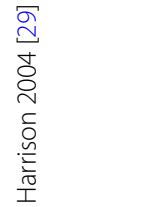 & 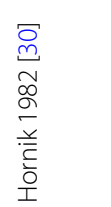 & 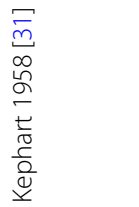 & 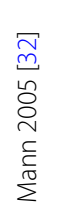 & 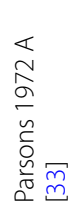 & 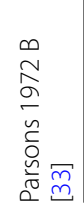 \\
\hline
\end{tabular}




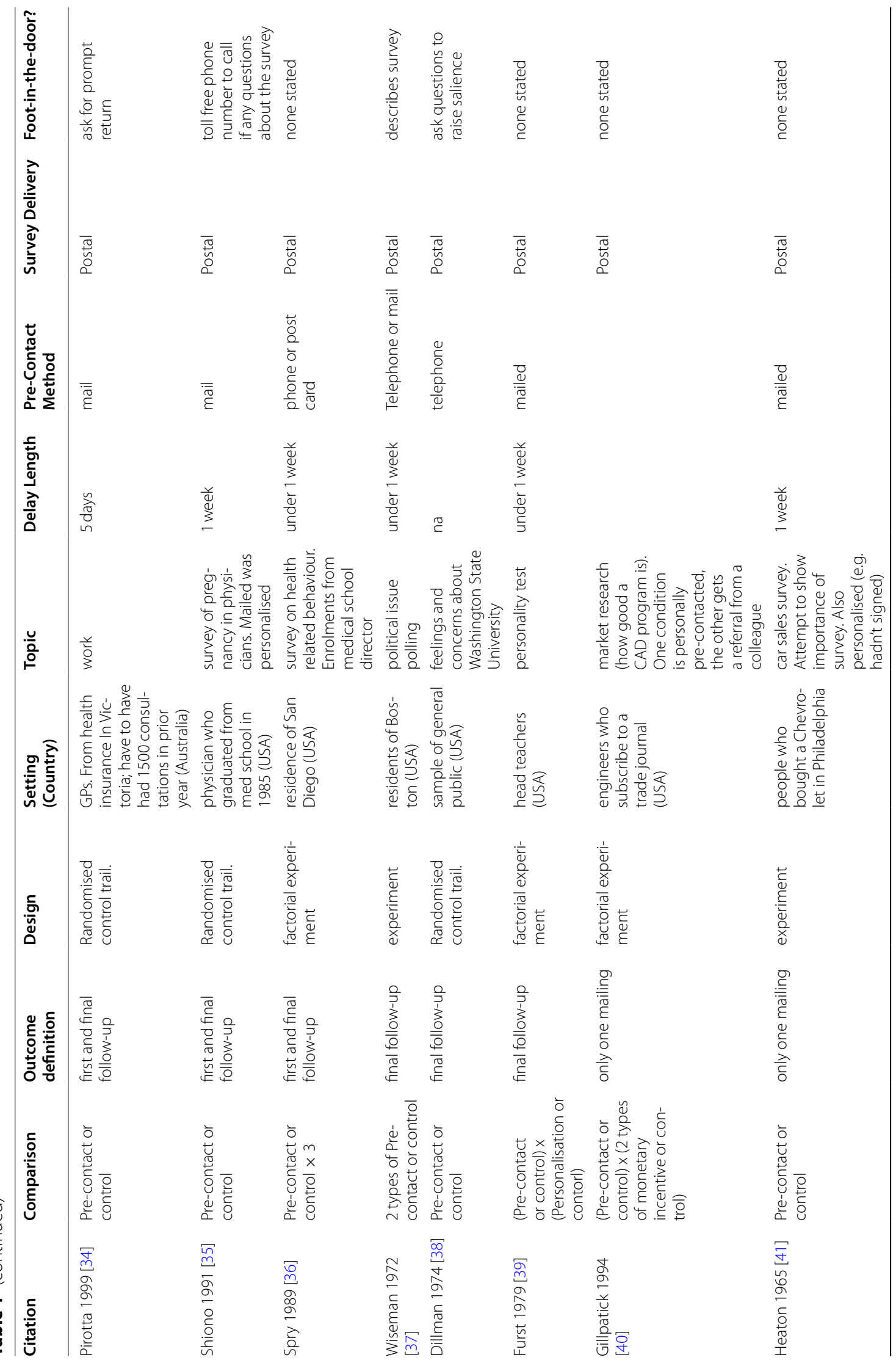




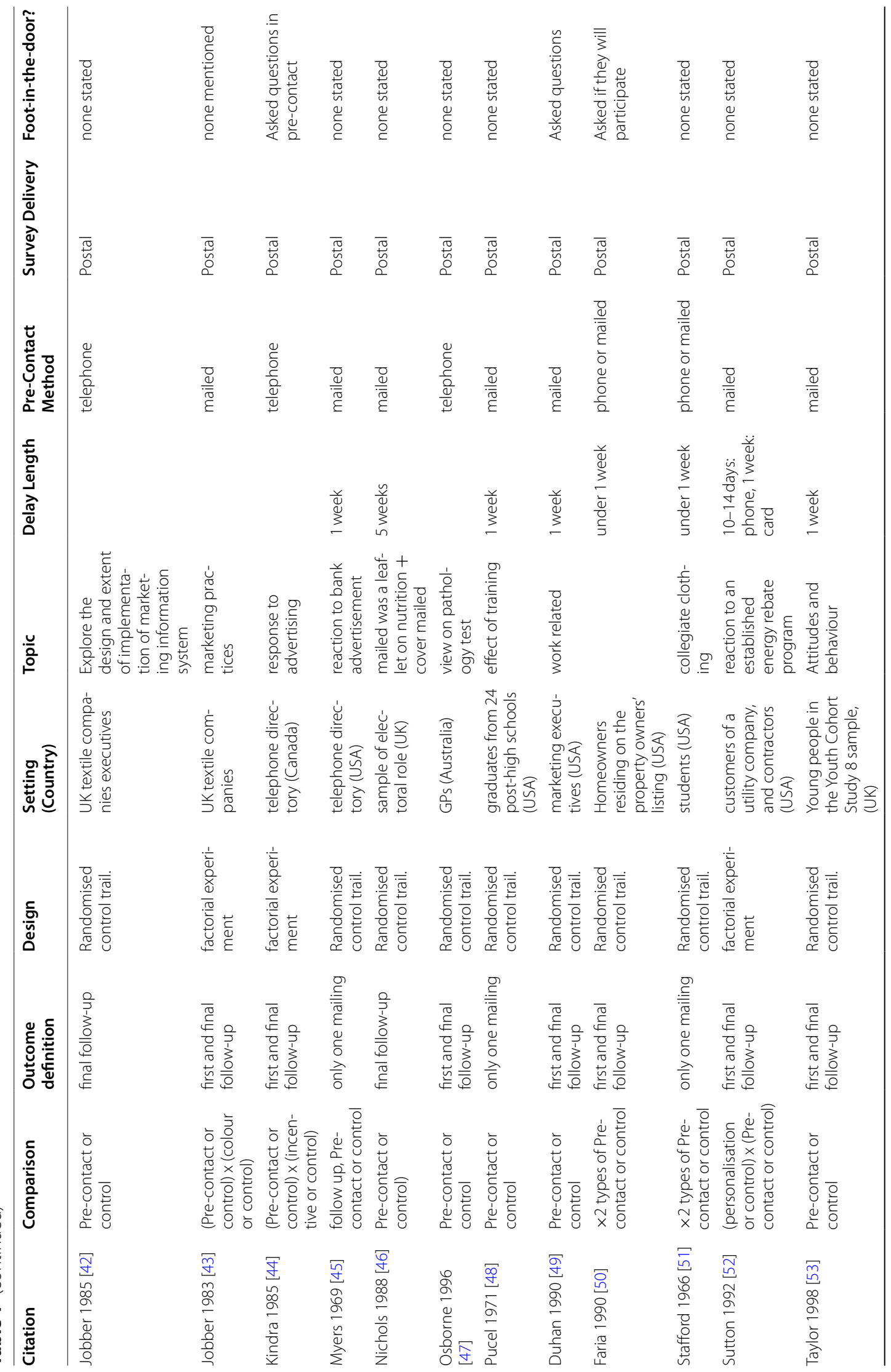




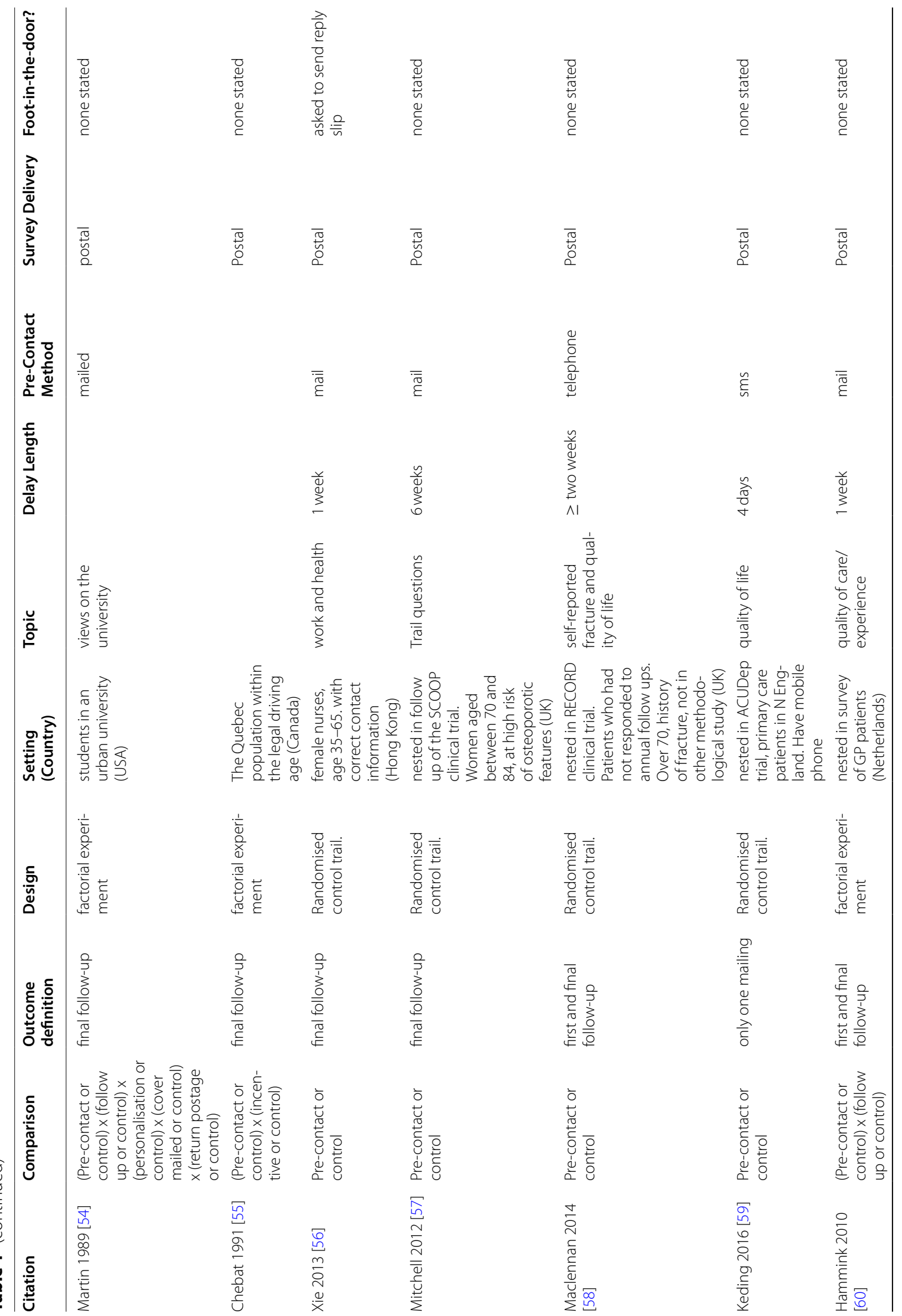




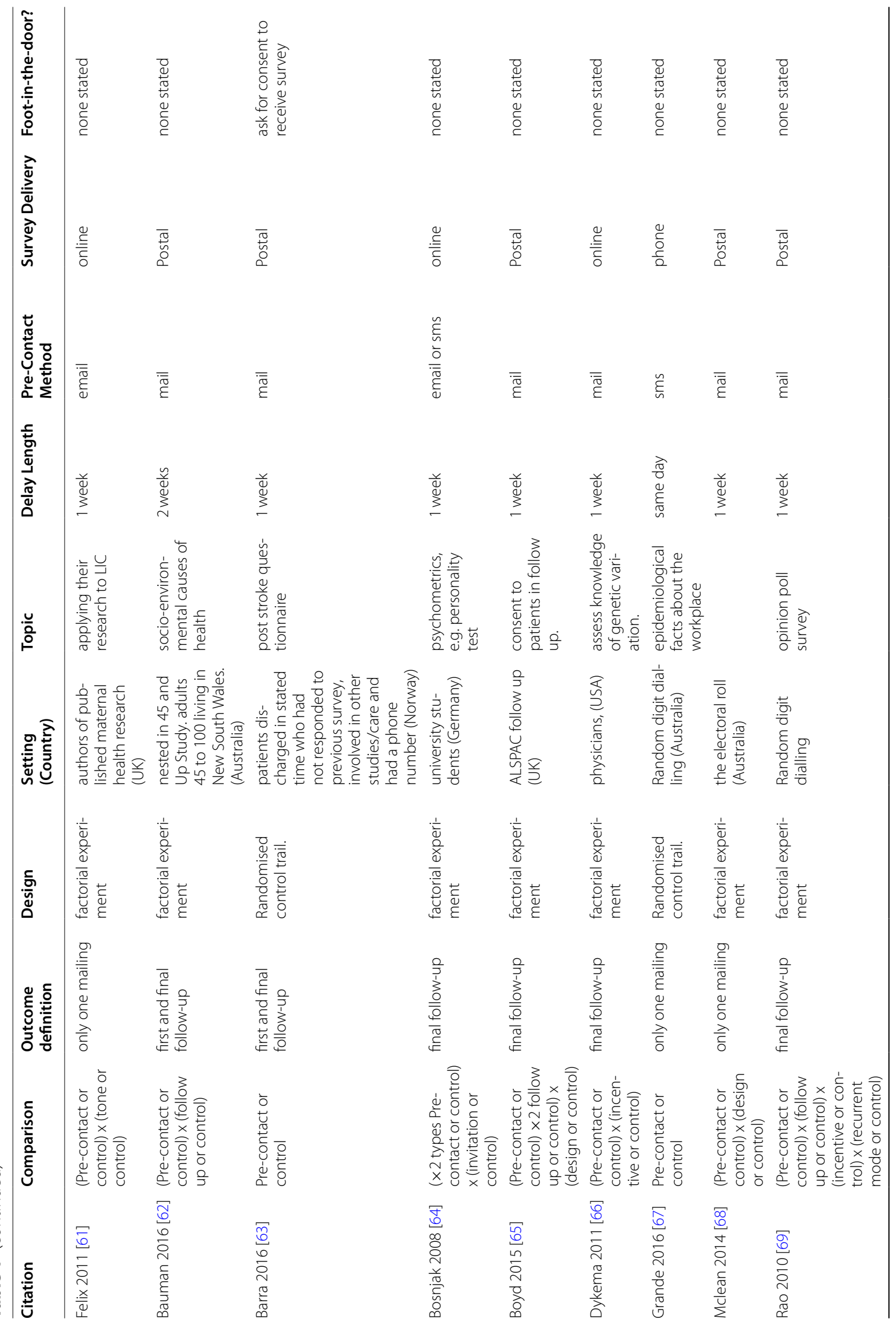




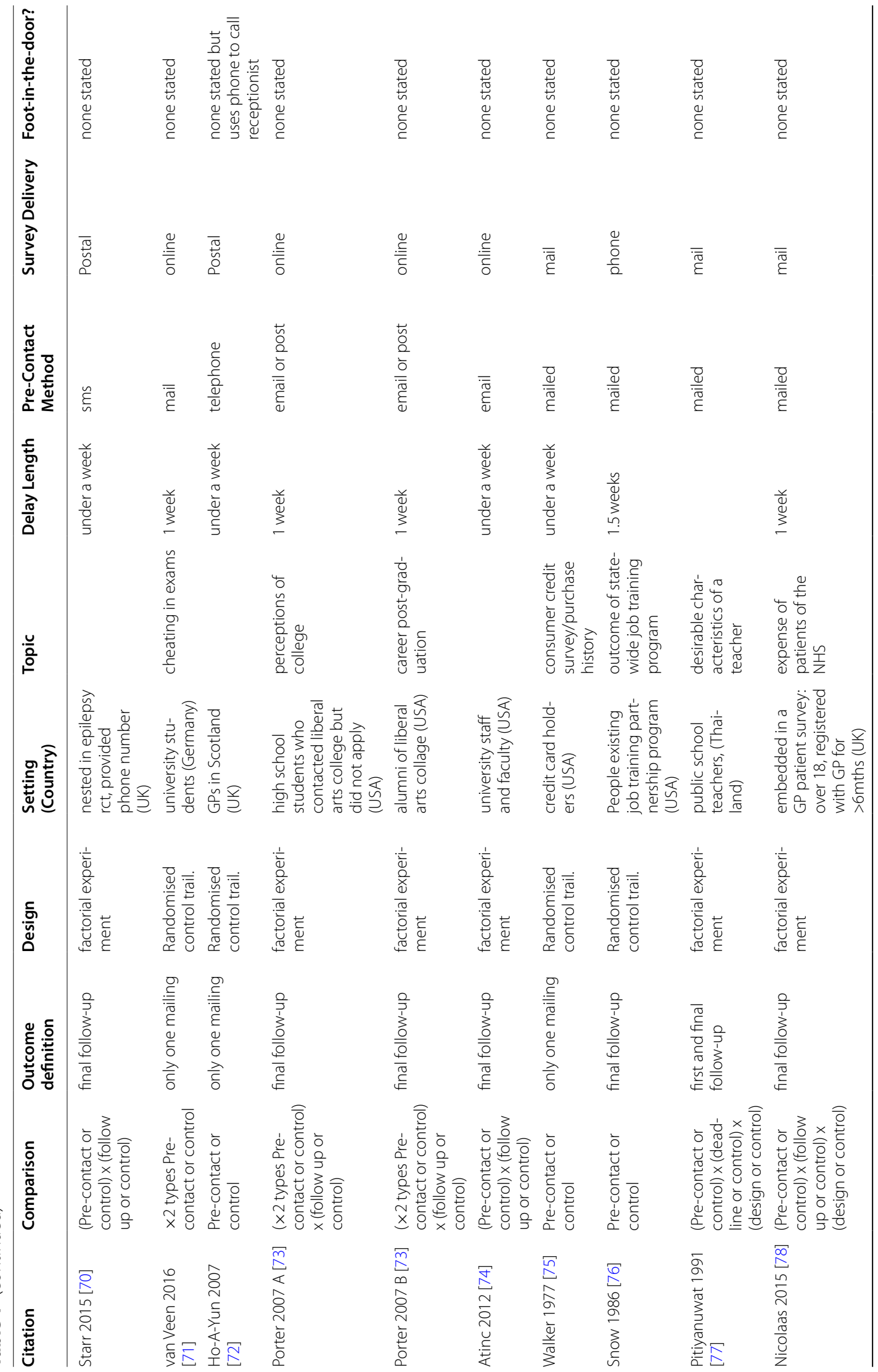




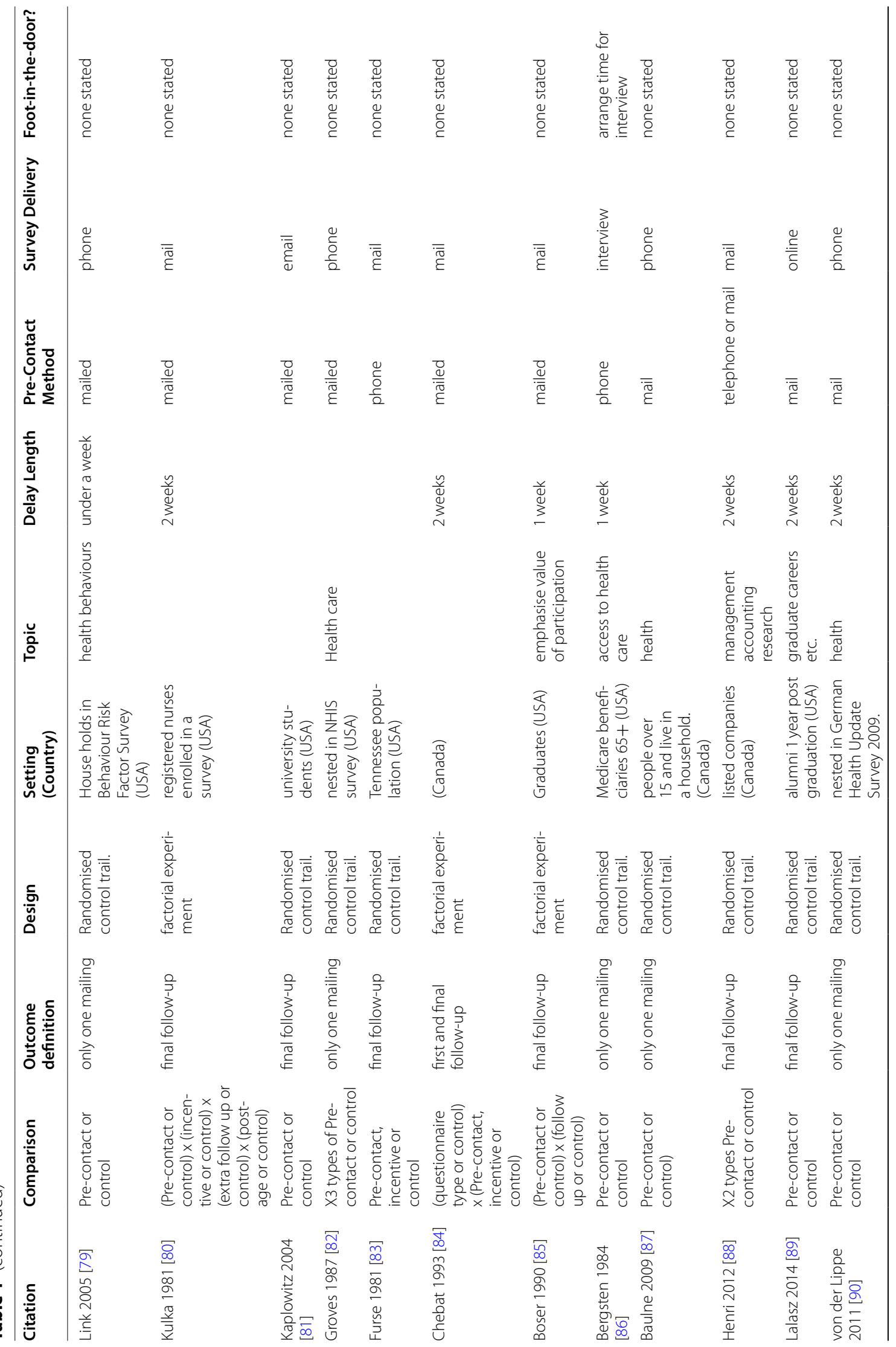




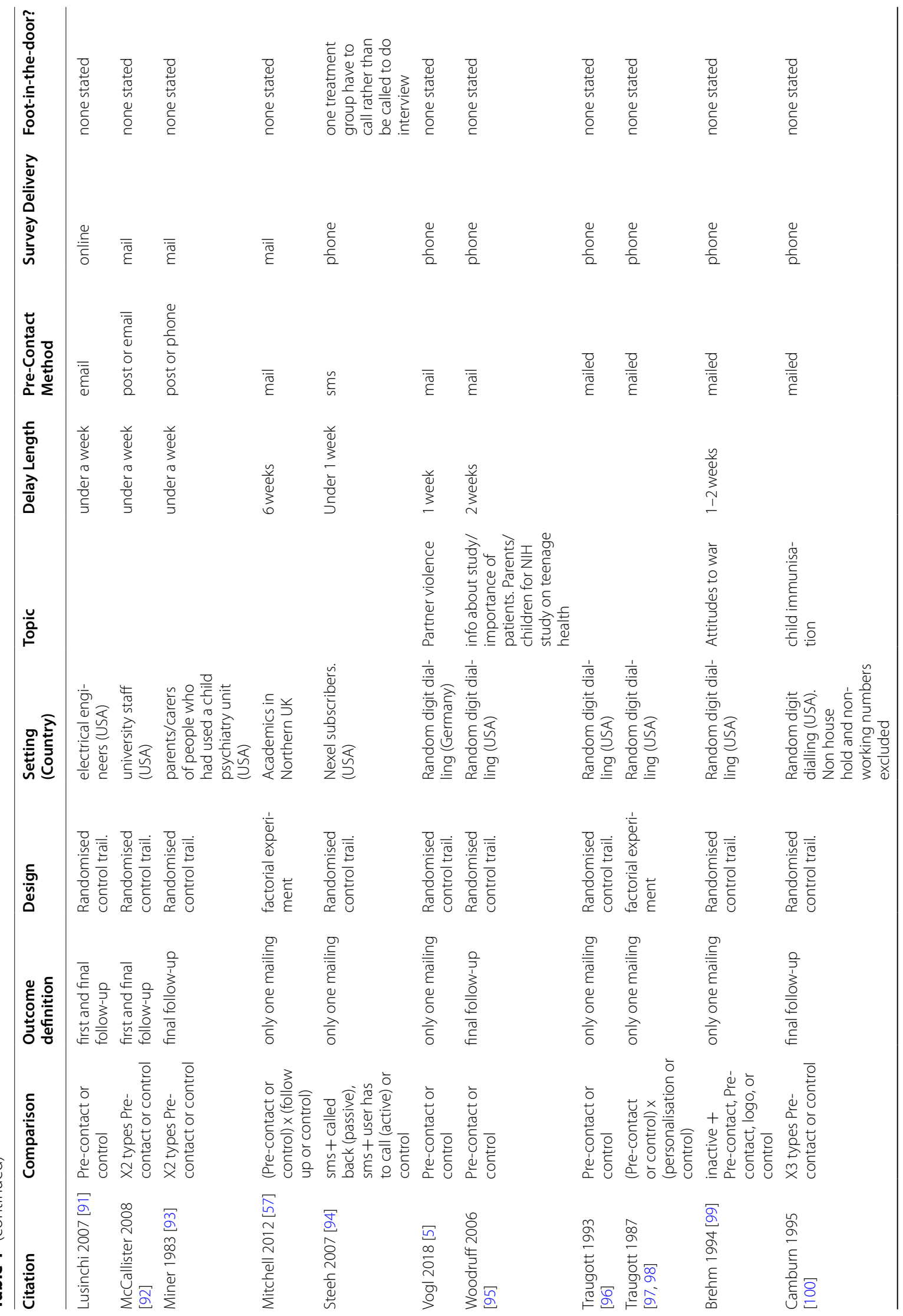




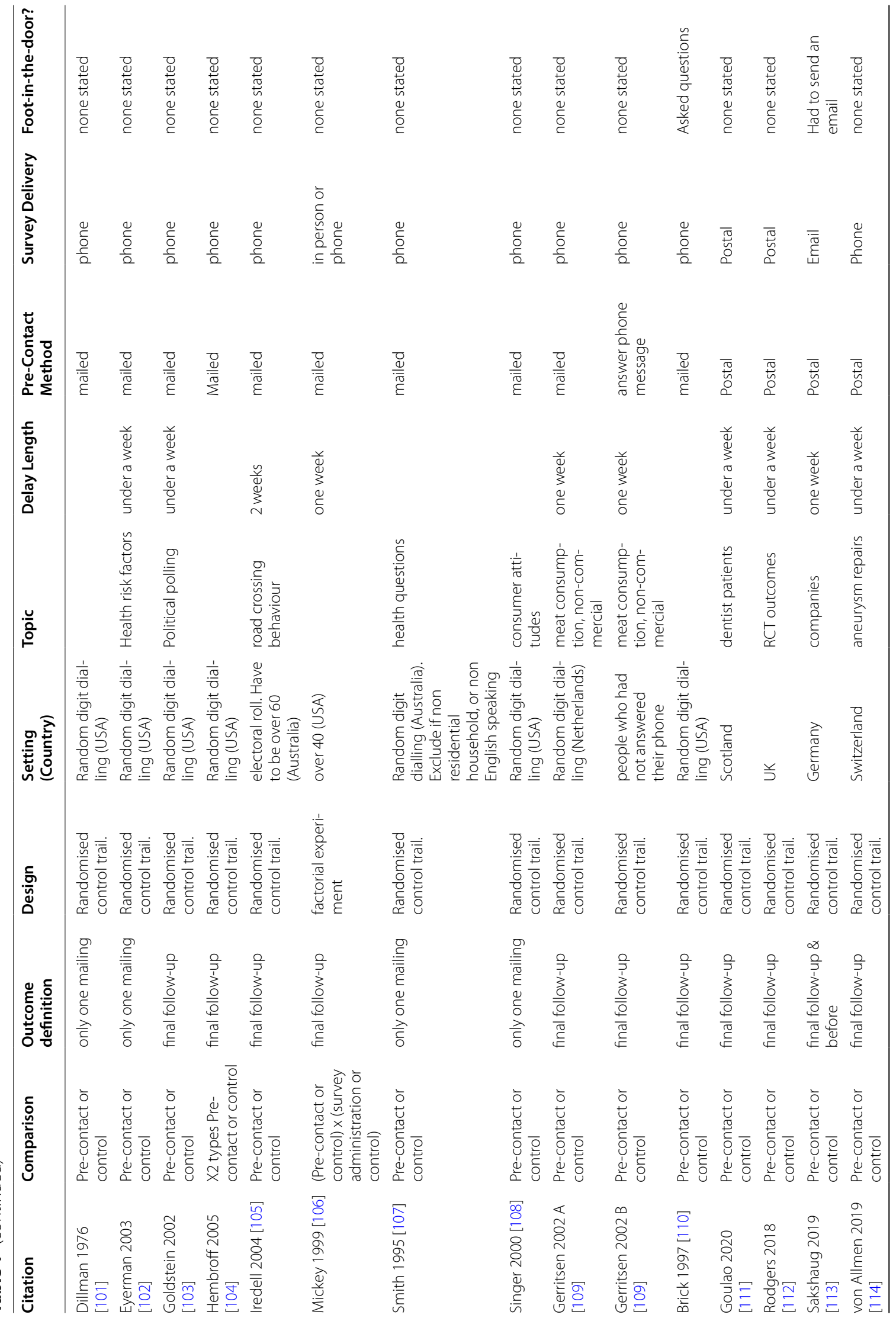




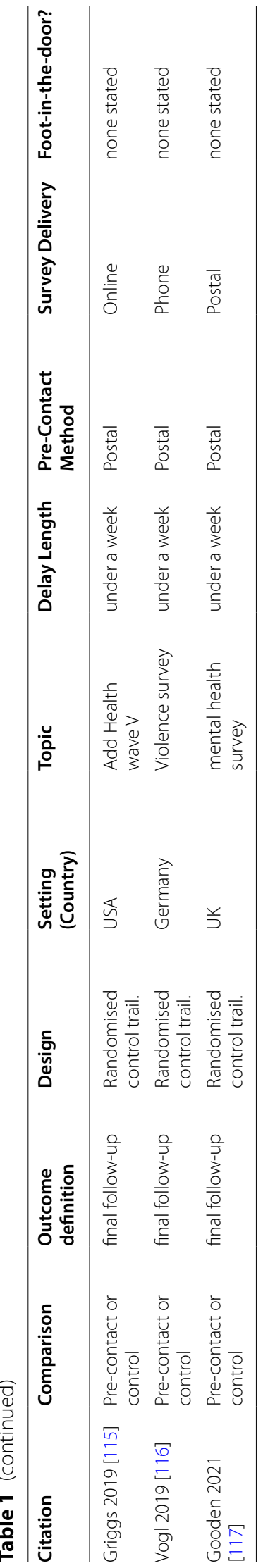




\section{Risk of bias within studies}

Judgments formed for each domain of the Cochrane Risk of Bias tool in each study are represented graphically in Fig. 2. The supporting evidence can be found in Supplementary Table 6. Overall, 8 studies were at high risk, 21 at low risk and 78 were at unclear risk. The proportions of studies at each level of risk is presented in Fig. 3.

\section{Sequence generation}

Thirty-three studies described the process used to generate the random sequence, or confirmed the use of randomisation in correspondence. Seventy-four studies have an uncertain risk of bias.

\section{Allocation concealment}

Thirty studies described concealment, or confirmed it in communication. Five confirmed that they had not used allocation concealment in communication. The remaining 72 studies provided insufficient information to reach a judgment, and so are of unclear bias.

\section{Participant and personnel blinding}

Participant and personnel blinding was not reported most trials. However, the design of many trials ensured that a degree of blinding did occur. A common design was to randomise participants to receive or not to receive a pre-notification without prior consent. The pre-notification itself would also often not explain that the participant had been allocated to receive it randomly. Thus any effect of treatment could not be due to the effect of knowing that they had been specially selected for an intervention which others had not got. Although the participant still knew they had received the pre-notification, this knowledge is part of the effect of a pre-notification - and therefore does not introduce any risk of material bias.

Similarly, although most did not describe any blinding procedure for personnel, its absence was often unlikely to lead to bias in estimates. In studies using a pre-written pre-contact (e.g. e-mail, letters, SMS) unblinded study personnel do not have the ability to influence the experience or perceptions of potential participants, as their only means of communication with each other is through a pre-written pro-forma message. This, however, is not true for studies which used a telephone pre-notification, in which the personnel and potential participants can have a genuine interaction. No study with telephone prenotification reported no blinding of personnel.

Overall 92 studies were regarded as being at low risk of bias, and 15 at unclear risk.

\section{Blinding of outcome assessment}

Outcome assessment blinding was reported in 8 studies. However, the outcome (whether the questionnaire had been returned) is objective, and unlikely to be influenced by whether the outcome assessor knows the group assignment. Because the analyses are a comparison of two proportions, data analysers were unlikely to have enough researcher degrees of freedom for bias to be introduced in the analyses. All studies were therefore judged as being at low risk of bias for this domain.

\section{Incomplete outcome data}

One hundred three provided enough information to ascertain the total number of participants randomised in each arm and the total number of questionnaires returned in each arm. However, 4 are at unclear risk because they did not report sufficient detail to estimate per protocol rates, or state if the rates were intention to treat or per protocol, and one study at high risk.

\section{Selective reporting}

There was little evidence of selective reporting. All studies reported information on the relevant outcomes of interest. However, study protocols were not examined.

\section{Other biases}

Three of the factorial studies had significant interaction effects.

\section{Results of individual studies}

The results from individual studies are presented in a forest plot, Fig. 4. Fifty-nine studies had 95\% confidence intervals which were incompatible with the null hypothesis, of which 55 implied that pre-notification increased response rates. There were a number of studies which appeared to have extreme results (Stafford 1966 [51]; Kulka 1981 [80]; Gillpatick 1994 [40]; Rodgers 2018 [112]; Sakshaug 2019 [113]; Taylor 1998 [53]). The extreme result of Rodgers appears to be due to the unusually high overall rate of response $(97.1 \%)$. The other apparent outliers all were at high or unclear risk of bias.

\section{Synthesis of results}

Information on response was available in all trials, thus data from all trials was used. These randomised a total of 338,429 participants, and had 174,323 returned questionnaires. The pooled estimate shows an increase in response for the final follow-up after questionnaire prenotification ( $\mathrm{OR}=1.33,95 \% \mathrm{CI}: 1.20-1.47, p<0.001)$, compared to an increase of 1.45 (95\% CI 1.29 to 1.63 ) for Edwards 2009 (Supplementary Table 7). There was 


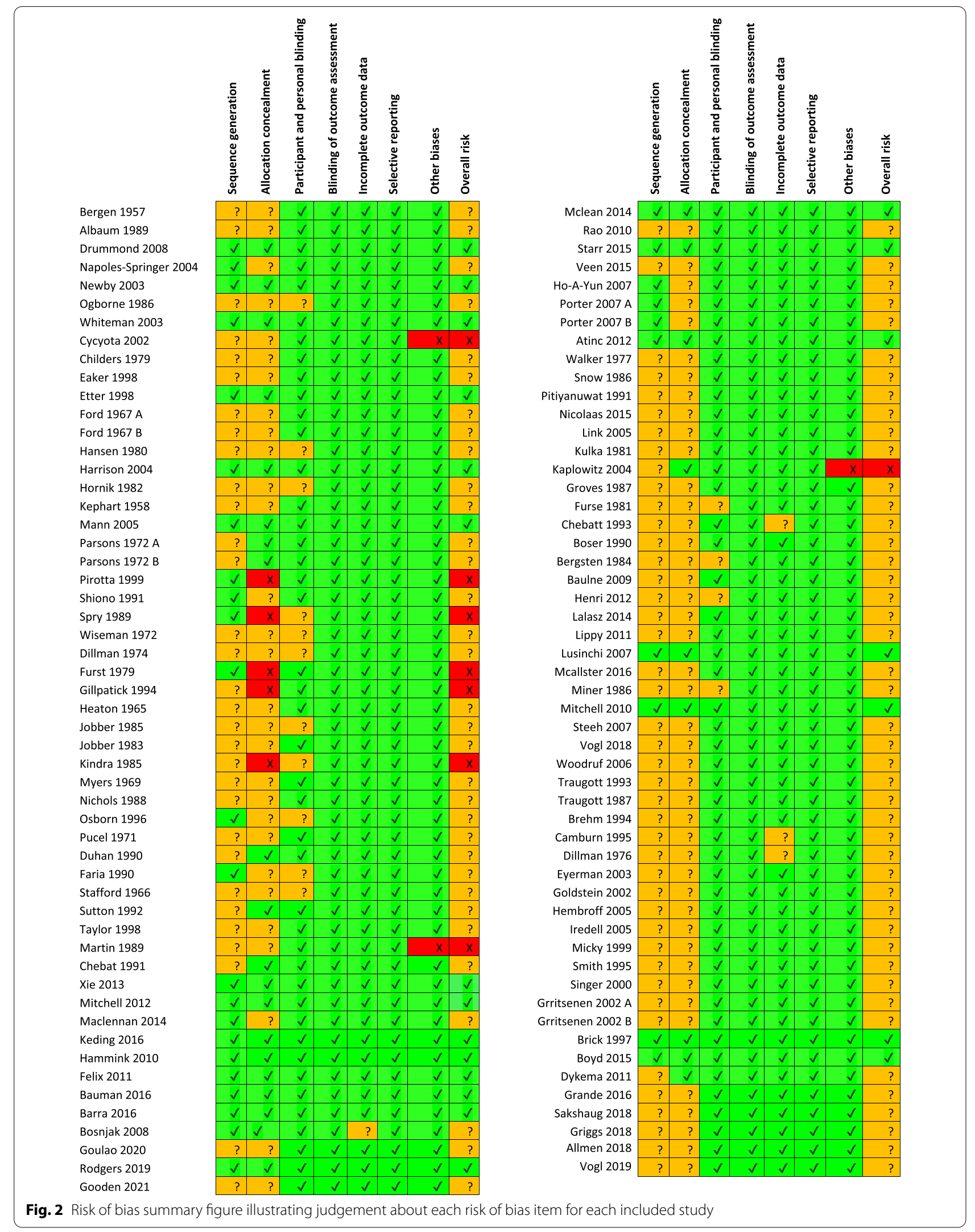




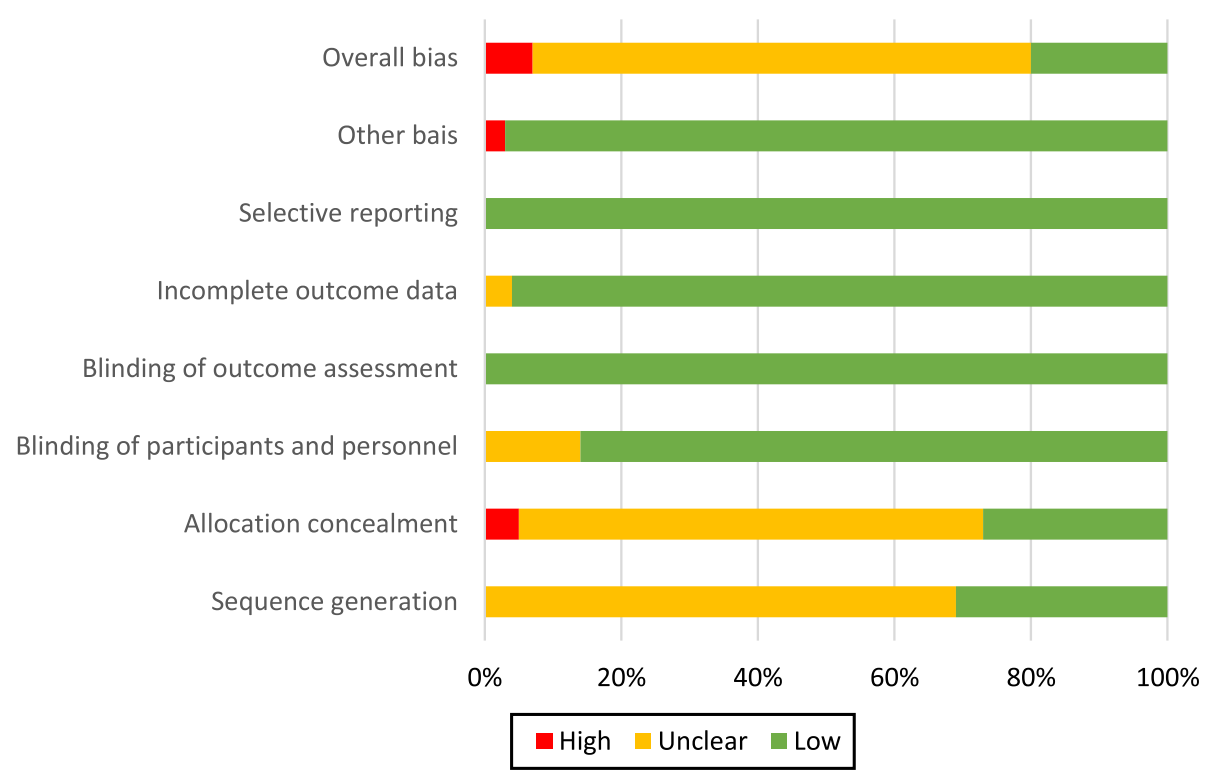

Fig. 3 Risk of bias graph illustration judgments about each risk of bias item presented as percentages across all included studies

strong evidence of heterogeneity $\left(\mathrm{I}^{2}=97.1 \%\right.$; $\mathrm{Tau}^{2}=$ $\left.0.26 ; \mathrm{X}^{2}(107, N=107)=3710.90, p<0.001\right)$.

All subgroups, in the stratified meta-analyse, show significant amounts of heterogeneity (Supplementary Table 8). However, studies with low risks of bias and which send the pre-notification online had 95\% confidence intervals which were compatible with the null hypothesis and appears to have reduced $\mathrm{I}^{2}$ (67.4 and $65.1 \%$ respectively).

\section{Risk of bias across studies}

To explore the possibility of small study bias, funnel plots were created for the outcome, Fig. 5. Visual assessment implies that there is no major asymmetry. However, more studies than expected fell outside the 95\% confidence limits. In addition, a formal assessment of asymmetry, using Harbord's test, did not find evidence to reject the null hypothesis of no asymmetry $(p=0.749)$.

\section{Effect of risk of bias within studies on the pooled results}

Seventy-eight studies were at unclear risk, 21 at low risk, and 8 at high. When stratified by risk of bias, there was no longer evidence against the assumption of a pooled association across studies which were of low bias $(\mathrm{OR}=1.09$, 95\% CI: 0.99-1.20, Fig. 6).

\section{Assessment of certainty in the evidence Risk of Bias}

Across domains, high risk of bias was uncommon. However, few studies provided sufficient information to be assigned low risk of bias. The interpretation of the overall results is therefore downgraded.

\section{Imprecision}

Due to the large number of participants in each arm, even after stratification by bias risk, confidence intervals were relatively narrow. GRADE suggests additionally assessing he 'optimum information size' (i.e. have the number of participants a randomised trial needs to have sufficient power to answer the question) $[118,119]$. Because larger sample sizes are required to detect smaller estimates, we calculated the optimum information size using information from the meta-analysis of studies at a low risk of bias (see Supplementary Table 7). Around 2500 participants would be required for each arm, for a $90 \%$ power and $5 \%$ alpha, which was obtained for both estimates.

\section{Indirectness}

There was generally little indirectness in the review. All studies were randomised control trials examining the effect of pre-notification on questionnaire response, so directly answered the review's question.

\section{Publication bias}

Visual inspection of the funnel plots and formal testing with Harbord's test both imply that small study bias was unlikely. As high questionnaire response is important to non-academics, e.g. polling companies, an unassessed grey literature will probably exist. 


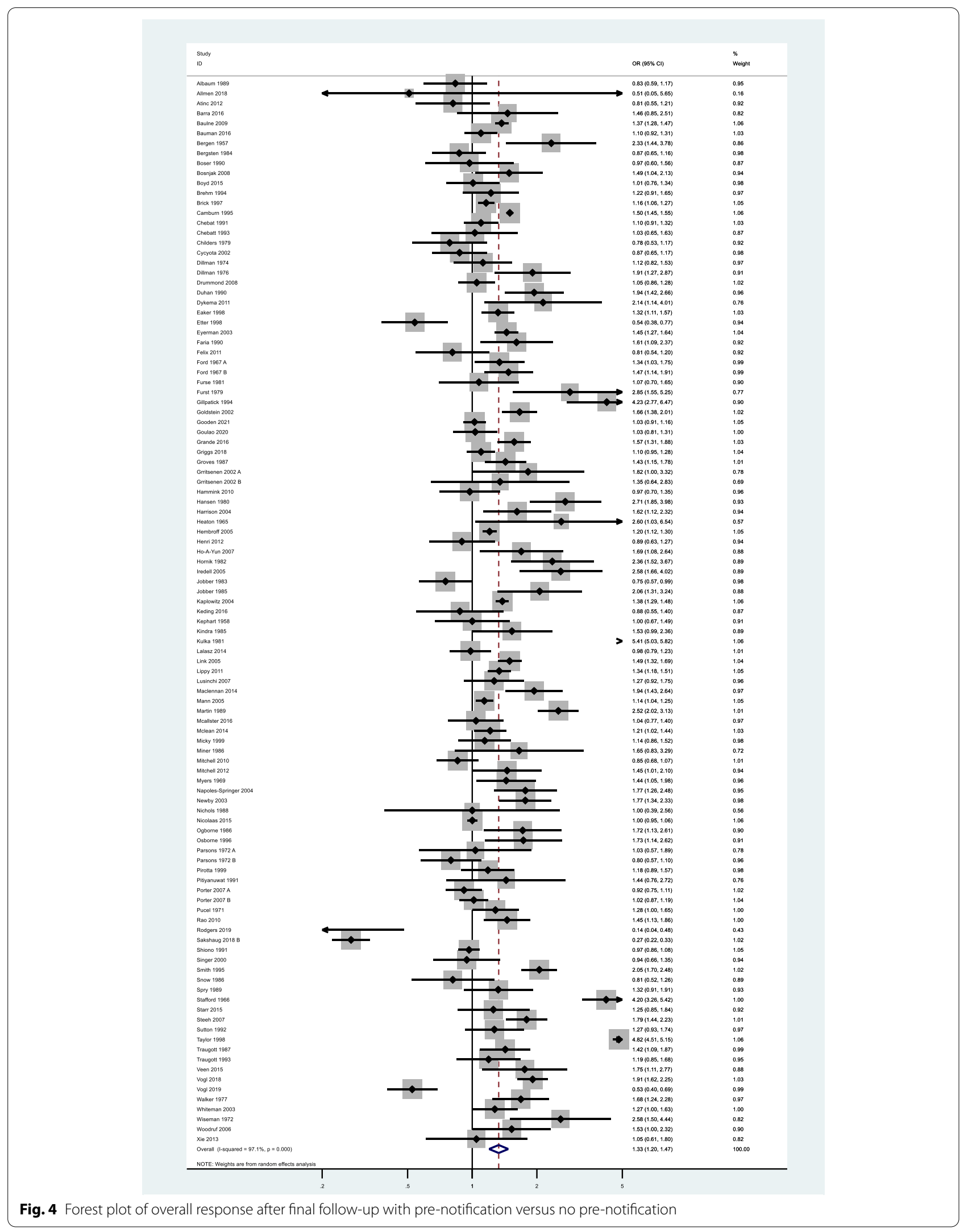




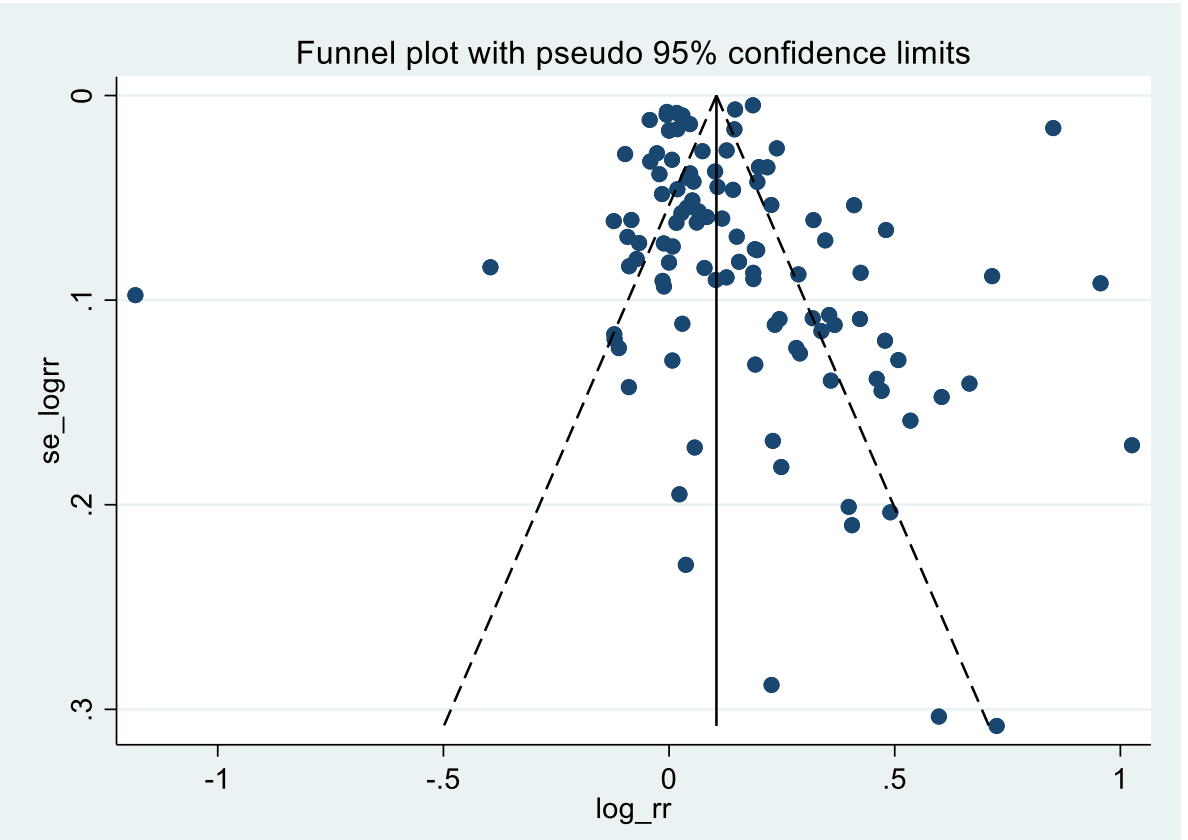

Fig. 5 Funnel plot with pseudo 95\% confidence limits for response after final follow-up

\section{Heterogeneity}

There was substantive heterogeneity within the review, and in all stratified analyses. We therefore downgraded the evidence due to the unexplained heterogeneity. Future studies should consider further explanations.

\section{Overall GRADE evaluation}

After two downgrades, there is low certainty in the overall estimate, but, with only one downgrade, moderate certainty in the estimate for studies at low risk of bias.

\section{Discussion and conclusions}

\section{Summary and interpretation of evidence}

This meta-analysis and systematic review of randomised control trials examined the effect of pre-notification compared to no pre-notification on questionnaire response rates. Pre-notification led to 1.33 (95\% CI: $1.20-$ 1.47) times greater odds for response. However, this was greatly reduced after restricting to studies of low risk of bias, OR = 1.09 (95\% CI: 0.99-1.20).

This low OR implies that researchers should be cautious when using pre-notification as they may not lead to improvements in participant response rates. Specifically, in instances where pre-notification would be an expensive addition to a study, we believe that there is too much uncertainty to recommend the use of a pre-notification. One potential implication of the remaining unexplained heterogeneity is that there are unmeasured effect modifiers which cause pre-notification to work in some circumstances but not other. Therefore, if pre-notification would have a negligible impact on the cost of recruiting participants, nesting a high-quality randomised control trail could help reduce the uncertainty around the potential benefits of pre-notification in a specific setting.

\section{Limitations \\ Limitations of the evidence included in the review}

Level of certainty in the evidence The level of certainty in both the overall and low risk of bias estimates were downgraded because of high unexplained heterogeneity. Exploring other factors could be a topic of other reviews. The large number of high and unclear risk of bias studies lead to the overall estimate being downgraded an additional time.

The number of studies with an unclear risk of bias could have potentially been reduced if studies in the 2021 search were contacted for further information. However, the age of many of the remaining studies made communication difficult, e.g. due to address change, and information not being available for studies where contact could be made. In addition, between the beginning of the project and its end Cochrane released an updated version of the Risk of Bias tool. The new tool changed the structure 


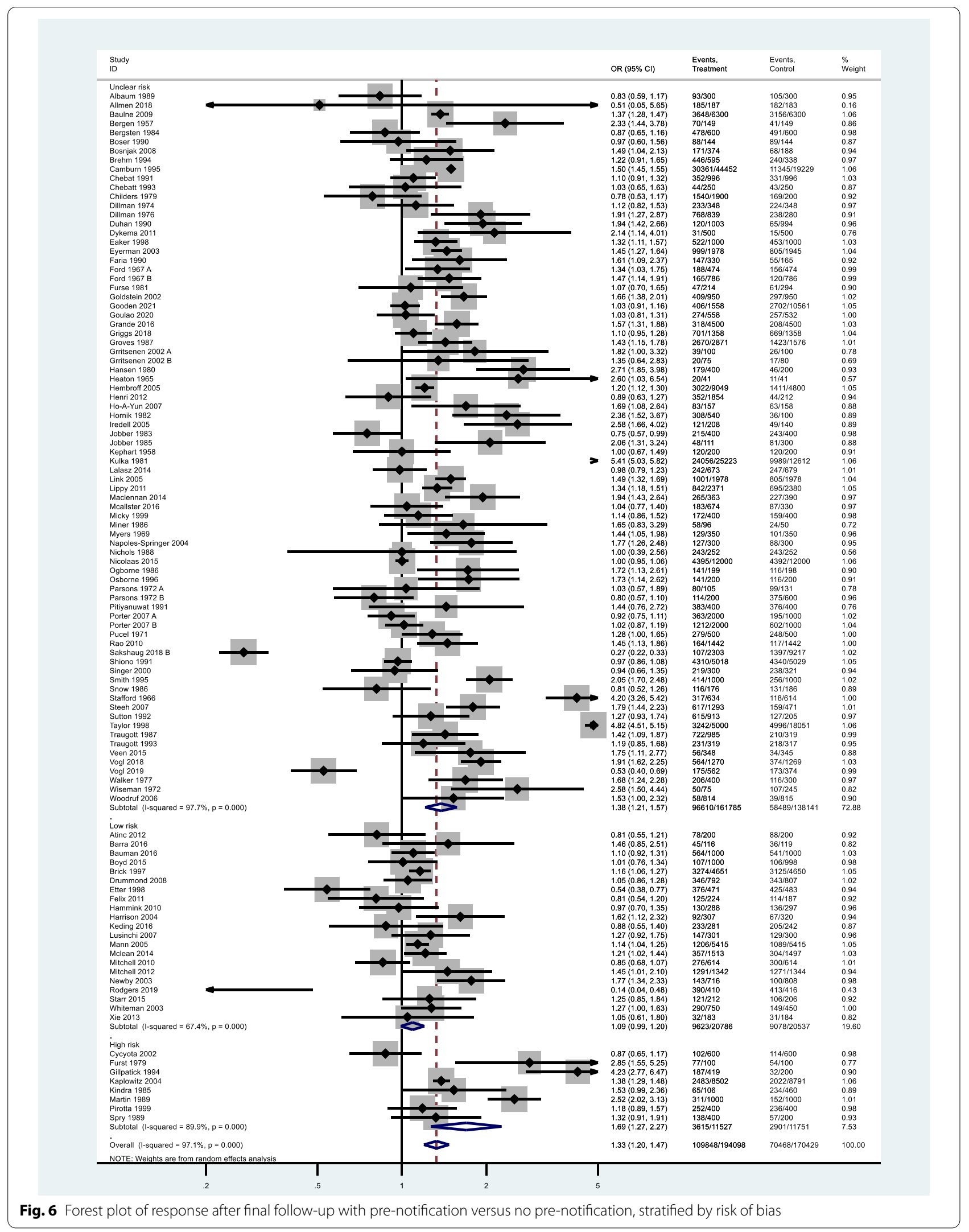


of the evaluation and by allows reviewers to come to a qualitative decision about the probability of bias risk in each domain. Most studies with an unclear risk of bias have it because they did not describe randomisation and/or allocation concealment in sufficient detail. It is likely that many of these studies could have been either upgraded or downgraded when evaluated using ROB2 based of covariate balance. We would therefore expect fewer studies to have an unclear risk of bias if we had used ROB2.

Generalisability There are very few studies from lowor middle-income countries. The review's results may not generalise to any population, especially given the heterogeneous effect.

\section{Limitations of the review process}

Search strategy Cochrane recommends that the literature searching be done by two independent reviewers, while this review only used one [120]. In addition, the search lacked specificity, and some extra publications might have been found by contacting authors to see if they had published other studies on the question. However, citation searching is not always common in systematic reviews, although it proved an effective way of detecting new studies.

Data extraction and risk of Bias assessment Cochrane recommends that data extraction should be done by two independent reviewers [121]. Although this review only used one reviewer to extract data and conducted the risk of bias assessment, both were done twice by this reviewer, which should also reduce transcription errors. There is still, however, some risk of bias due to the reviewer being unblinded.

\section{Strengths and weaknesses in relation to other studies}

The updated review more than doubled the number of included studies, even with four old studies were excluded for poor methodology (Supplementary Table 5). The overall results of the two studies are relatively similar, with overlapping confidence intervals overlap the results of the two studies might be consistent. However, restricting to low risk of bias studies implies that this estimate may be due to study bias. Therefore, while Edwards et al. (2009) concluded that pre-notification does improve response rates, this review would conclude that there is moderate evidence that pre-notification may not improve response rates to questionnaires.
Both Edwards et al., and this study, might be criticised for their choice of outcomes. Response rate does not entail response quality [5]. For example, a questionnaire might not have been fully completed, or completed inaccurately. In addition, to be a useful intervention for researchers pre-notification needs to be cost effective. However, neither of these outcomes are examined in the reviews.

The conclusion was also different from two other systematic reviews which explored a similar question. Both Lacy et al., and van Gelder et al., concluded that pre-notification did improve response rates (with $\mathrm{OR}=1.45$, 95CI 1.01 to 2.10 , and $\mathrm{OR}=1.12,95 \% \mathrm{CI} 1.12$ to 1.22 respectively) [122-165]. However, the $95 \%$ CI of both of these studies is compatible with the results of this study, and neither of these studies stratified their metanalyses by risk of bias.

\section{Conclusions and implications for further studies and practice}

This systematic review and meta-analyses of randomised control trials examining the effect of pre-notification on questionnaire response found evidence which supports the use of pre-notification. However, after excluding studies at high or unclear risk of bias the effect of the intervention was greatly reduced, and is probably no longer of relevance. The quality of evidence among low risk of bias studies was downgraded due to substantial unexplained heterogeneity. Future reviews could consider exploring other explanations. In addition, studies originated from a limited set of settings, such as generally high-income countries. Future studies could explore if the results generalise to new settings.

\section{Supplementary Information}

The online version contains supplementary material available at https://doi. org/10.1186/s12874-021-01435-2.

Additional file 1.

Additional file 2.

Additional file 3.

Additional file 4.

Additional file 5.

Additional file 6 .

Additional file 7.

Additional file 8.

Acknowledgments

Not applicable.

Future updates

At the time of writing, PE is planning a complete update of the 2009 review. 


\section{Authors' contributions}

BW designed the study, and PE supervised the project. BW implemented the design and wrote the manuscript. PE provided feedback, and checked the screening quality. The authors read and approved the final manuscript.

\section{Funding}

Benjamin Woolf is funded by an Economic and Social Research Council (ESRC) South West Doctoral Training Partnership (SWDTP) $1+3$ PhD Studentship Award (ES/P000630/1).

\section{Availability of data and materials}

Data and materials not available in the paper will be made available though contacting the corresponding author.

\section{Declarations}

\section{Ethics approval and consent to participate}

NA.

\section{Consent for publication}

Not applicable.

\section{Competing interests}

The authors declare they have no competing interests.

\section{Author details}

${ }^{1}$ Department of Psychological Science, University of Bristol, 5 Priory Road, Bristol, UK. ${ }^{2}$ Medical Research Council Integrative Epidemiology Unit, University of Bristol, Bristol, UK. ${ }^{3}$ Faculty of Epidemiology and Population Health, London School of Hygiene and Tropical Medicine, London, UK.

Received: 13 April 2021 Accepted: 11 October 2021

Published online: 27 November 2021

\section{References}

1. Gelder VJMMH, Bretveld RW, Roeleveld N. Web-based questionnaires: the future in epidemiology? Am J Epidemiol. 2010;172(11):1292-8.

2. Doliński D. Is psychology still a science of behaviour? Soc Psychol Bull. 2018;13(2):e25025.

3. Van der Stede WA, Young SM, Chen CX. Assessing the quality of evidence in empirical management accounting research: the case of survey studies. Acc Organ Soc. 2005;30(7):655-84

4. Scott P, SPMF. Epidemiology for Canadian Students: Principles, Methods and Critical Appraisal. 1st ed: Brush Education; 2015. p. 304

5. Vogl S. Advance Letters in a Telephone Survey on Domestic Violence: Effect on Unit Nonresponse and Reporting. Int J Public Opin Res. [cited 2018 Aug 29]; Available from: https://academic.oup.com/ijpor/advan ce-article/doi/10.1093/ijpor/edy006/4944573.

6. Edwards PJ, Roberts I, Clarke MJ, Diguiseppi C, Wentz R, Kwan I, et al. Methods to increase response to postal and electronic questionnaires. Cochrane Database Syst Rev. 2009;3:MR000008.

7. Page MJ, McKenzie JE, Bossuyt PM, Boutron I, Hoffmann TC, Mulrow CD, et al. The PRISMA 2020 statement: an updated guideline for reporting systematic reviews. BMJ. 2021;372:n 71. https://doi.org/10.1136/bmj. n71.

8. Higgins JPT, Altman DG, Sterne JAC. Chapter 8: Assessing risk of bias in included stuides. In: Higgins JPT, Green S, editors. Cochrane Handbook for Systematic Reviews of Interventions. Version 5.2.0 [updated June 2017]: The Cochrane Collaboration; 2017. Available from www.coch ane-handbook.org.

9. Higgins JPT, Deeks JJ. Chapter 16: Speical topics in statistics. In: Higgins JPT, Green S, editors. Cochrane Handbook for Systematic Reviews of Interventions. Version 5.1.0 [updated March 2011]: The Cochrane Collaboration; 2011. Available from www.cochrane-handbook.org.

10. Sterne JAC, Egger M, Moher D, Boutron I. Chapter 10: addressing reporting biases. In: Higgins JPT, Churchill R, Chandler J, Cumpston MS, editors. Cochrane handbook for systematic reviews of interventions version 5.2.0 (updated June 2017), Cochrane; 2017. Available from www.training.cochrane.org/handbook.

11. Siemieniuk R, Guyatt G. What is GRADE. BMJ J Best Practi. 2019;10.

12. Temple-Smith M, Mulvey G, Doyle W. Maximising response rates in a survey of general practitioners - lessons from a Victorian survey on sexually transmissible diseases. Aust Fam Physician. 1998;27(Suppl 1):S15-8.

13. Waisanen FB. A note on the response to a mailed questionnaire. Public Opin Q. 1954;18:210-2.

14. Wright M. The effect of pre-notification on mail survey response rates: an experimental result. Mark Bull. 1995;6:59-64.

15. Wynn GW, McDaniel SW. The effect of alternative foot-in the-door manipulations on mailed questionnaire response rate and quality. J Mark Res Soc. 1985;27(1):15-26.

16. Bergen AV, Spitz JC. De introductie van een schriftelijke enquete. Ned Tijdschr Psychol. 1957;12:68-96.

17. Albaum G, Strandskov J. Participation in a mail survey of international marketers: effects of pre-contact and detailed project explanation. J Glob Mark. 1989;2(4):7-23.

18. Drummond FJ, Sharp L, Carsin AE, Kelleher T, Comber H. Questionnaire order significantly increased response to a postal survey sent to primary care physicians. J Clin Epidemiol. 2008;61:177-85.

19. Napoles-Springer AM, Fongwa MN, Stewart AL, Gildengorin G, PerezStable EJ. The effectiveness of an advance notice letter on the recruitment of African Americans and whites for a mailed patient satisfaction survey. J Aging Health. 2004;16(5 Suppl):124S-36S.

20. Newby R, Watson J, Woodliff D. SME survey methodology: Response rates, data quality, and cost effectiveness. Entrep Theory Pract. 2003;28(2):163-72.

21. Ogbourne AC, Rush B, Fondacaro R. Dealing with nonrespondents in a mail survey of professionals. Eval Health Prof. 1986;9(1):121-8.

22. Whiteman MK, Langenberg P, Kjerulff K, McCarter R, Flaws JA. A randomized trial of incentives to improve response rates to a mailed women's health questionnaire. J Women's Health. 2003;12(8):821-8.

23. Cycyota C, Harrison DA. Enhancing survey response rates at the executive level: are employee- or consumer-level techniques effective? J Manag. 2002;28(2):151-76.

24. Childers TL, Skinner SJ. Gaining respondent cooperation in mail surveys through prior commitment. Public Opin Q. 1979;43:558-61.

25. Eaker S, Bergstrom R, Bergstrom A, Hans-Olov A, Nyren O. Response rate to mailed epidemiologic questionnaires: a population-based randomized trial of variations in design and mailing routines. Am J Epidemiol. 1998;147(1):74-82.

26. Etter J-F, Perneger TV, Laporte J-D. Unexpected effects of a prior feedback letter and a professional layout on the response rate to a mail survey in Geneva. J Epidemiol Community Health. 1998;52:128-9.

27. Ford NM. The advance letter in mail surveys. J Mark Res. 1967;4:202-4.

28. Hansen RA, Robinson LM. Testing the effectiveness of alternative footin-the-door manipulations. J Mark Res. 1980;17:359-64.

29. Harrison RA, Cock D. Increasing response to a postal survey of sedentary patients - a randomised controlled trial. BMC Health Serv Res. 2004:4(31):1-5.

30. Hornik J. Impact of pre-call request form and gender interaction on response to a mail survey. J Mark Res. 1982;19:144-51.

31. Kephart WM, Bressler M. Increasing the response to mail questionnaires: a research study. Public Opin Q. 1958;21:123-32.

32. Mann CB. Do advance letters improve preelection forecast accuracy? Public Opin Q. 2005;69(4):561-71.

33. Parsons RJ, Medford TS. The effect of advance notice in mail surveys of homogeneous groups. Public Opin Q. 1972;36:258-9.

34. Pirotta M, Gunn J, Farish S, Karabatsos G. Primer postcard improves postal survey response rates. Aust N Z J Public Health. 1999;23(2):196-7.

35. Shiono $\mathrm{PH}$, Klebanoff MA. The effect of two mailing strategies on the response to a survey of physicians. Am J Epidemiol. 1991;134(5):539-42.

36. Spry VM, Hovell MF, Sallis JG, Hofsteter CR, Elder JP, Molgaard CA. Recruiting survey respondents to mailed surveys: controlled trials of incentives and prompts. Am J Epidemiol. 1989;130(1):166-72.

37. Wiseman F. Methodological bias in public opinion surveys. Public Opin Q. 1972:36:105-8.

38. Dillman DA, Frey JH. Contribution of personalization to mail questionnaire response as an element of a previously tested method. J Appl Psychol. 1974;59(3):297-301. 
39. Furst $L G$, Blitchington WP. The use of a descriptive cover letter and secretary pre-letter to increase response rate in a mailed survey. Pers Psychol. 1979;32:155-9.

40. Gillpatick TR, Harmon RR, Tseng LP. The effect of a nominal monetary gift and different contacting approaches on mail survey response among engineers. IEE Trans Eng Manage. 1994;41:285-90.

41. Heaton E. Increasing mail questionnaire returns with a preliminary letter. J Advert Res. 1965;5:36-9.

42. Jobber D, Sanderson S. The effect of two variables on industrial mail survey returns. Ind Mark Manag. 1985;14:119-21.

43. Jobber D, Sanderson S. The effects of a prior letter and coloured questionnaire paper on mail survey response rates. J Mark Res Soc. 1983;25(4):339-49.

44. Kindra GS, McGown KL, Bougie M. Stimulating responses to mailed questionnaires. An experimental study. Int J Res Mark. 1985;2:219-35.

45. Myers JH, Haug AF. How a preliminary letter affects mail survey returns and costs. J Advert Res. 1969;9(3):37-9.

46. Nichols S, Waters WE, Woolaway M, Hamilton-Smith MB. Evaluation of the effectiveness of a nutritional health education leaflet in changing public knowledge and attitudes about eating and health. J Hum Nutr Diet. 1988;1:233-8.

47. Osborne MO, Ward J, Boyle C. Effectiveness of telephone prompts when surveying general practitioners: a randomised trial. Aust Fam Physician. 1996;25(1):S41-3.

48. Pucel DJ, Nelson HF, Wheeler DN. Questionnaire followup returns as a function of incentives and responder characteristics. Vocational Guid Q. 1971;1:188-93.

49. Duhan DF, Wilson RD. Prenotification and industrial survey responses. Ind Mark Manag. 1990;19:95-105.

50. Faria AJ, Dickinson JR, Filipic TV. The effect of telephone versus letter prenotification on mail survey response rate, speed, quality and cost. J Mark Res Soc. 1990;32(4):551-68.

51. Stafford JE. Influence of preliminary contact on mail returns. J Mark Res. 1966;3:410-1.

52. Sutton RJ, Zeitz LL. Multiple prior notifications, personalization, and reminder surveys. Mark Res. 1992:4:14-21.

53. Taylor S, Lynn P. The effect of a preliminary notification letter on response to a postal survey of young people. J Market Res Soc. 1998;40(2):165-73.

54. Martin WS, Duncan WJ, Powers TL, Sawyer JC. Costs and benefits of selected response inducement techniques in mail survey research. J Bus Res. 1989:19:67-79.

55. Chebat J-C, Picard J. Does prenotification increase response rates in mail surveys? A self-perception approach. J Soc Psychol. 1991;13(4):477-81.

56. Xie Y, Ho SC. Prenotification had no additional effect on the response rate and survey quality: a randomized trial. J Clin Epidemiol. 2013;66(12):1422-6

57. Mitchell N, Hewitt CE, Lenaghan E, Platt E, Shepstone L, Torgerson DJ, Scoop Study Team. Prior notification of trial participants by newsletter increased response rates: a randomized controlled trial. J Clin Epidemiol. 2012;65(12):1348-52.

58. MacLennan G, McDonald A, McPherson G, Treweek S, Avenell A Advance telephone calls ahead of reminder questionnaires increase response rate in non-responders compared to questionnaire reminders only: the RECORD phone trial. Trials. 2014;15:13

59. Keding A, Brabyn S, MacPherson H, Richmond SJ, Torgerson DJ. Text message reminders to improve questionnaire response rates. J Clin Epidemiol. 2016;79:90-5.

60. Hammink A, Giesen P, Wensing M. Pre-notification did not increase response rate in addition to follow-up: a randomized trial. J Clin Epidemiol. 2010;63(11):1276-8.

61. Felix LM, Burchett HE, Edwards PJ. Factorial trial found mixed evidence of effects of pre-notification and pleading on response to web-based survey. J Clin Epidemiol. 2011;64(5):531-6.

62. Bauman A, Phongsavan P, Cowle A, Banks E, Jorm L, Rogers K, et al. Maximising follow-up participation rates in a large scale 45 and up study in Australia. Emerg Themes Epidemiol. 2016;13:6.

63. Barra M, Simonsen TB, Dahl FA. Pre-contact by telephone increases response rates to postal questionnaires in a population of stroke patients: an open ended randomized controlled trial. BMC Health Serv Res. 2016;16(1):506.

64. Bosnjak M, Neubarth W, Couper MP, Bandilla W, Kaczmirek L. Prenotification in web-based access panel surveys: The influence of mobile text messaging versus e-mail on response rates and sample composition. Soc Sci Comput Rev. 2008;26(2):213-23.

65. Boyd A, Tilling K, Cornish R, Davies A, Humphries K, Macleod J. Professionally designed information materials and telephone reminders improved consent response rates: evidence from an RCT nested within a cohort study. J Clin Epidemiol. 2015;68(8):877-87.

66. Dykema J, Stevenson J, Day B, Sellers SL, Bonham VL. Effects of incentives and prenotification on response rates and costs in a national web survey of physicians. Eval Health Prof. 2011;34(4):434-47.

67. Grande ED, Chittleborough CR, Campostrini S, Dollard M, Taylor AW. Pre-survey text messages (SMS) improve participation rate in an Australian Mobile telephone survey: an experimental study. PLoS One. 2016;11(2):e0150231.

68. McLean SA, Paxton SJ, Massey R, Mond JM, Rodgers B, Hay PJ. Prenotification but not envelope teaser increased response rates in a bulimia nervosa mental health literacy survey: A randomized controlled trial. J Clin Epidemiol. 2014;67(8):870-6.

69. Rao K, Kaminska O, McCutcheon AL. Recruiting probability samples for a multi-mode research panel with internet and mail components. Public Opin Q. 2010;74(1):68-84.

70. Starr K, MCPherson G, Forrest M, Cotton SC. SMS text pre-notification and delivery of reminder e-mails to increase response rates to postal questionnaires in the SUSPEND trial: a factorial design, randomised controlled trial. Trials. 2015;16 Available from: https://www.ncbi.nlm.nih. gov/pmc/articles/PMC4494692/.

71. van Veen F, Göritz AS, Sattler S. Response Effects of Prenotification, Prepaid Cash, Prepaid Vouchers, and Postpaid Vouchers: An Experimental Comparison. Soc Sci Comput Rev. 2016;34(3):333-46.

72. Ho-A-Yun J, Crawford F, Newton J, Clarkson J. The effect of advance telephone prompting in a survey of general dental practitioners in Scotland: a randomised controlled trial. Community Dent Health. 2007;24(4):233-7.

73. Porter SR, Whitcomb ME. Mixed-mode contacts in web SurveysPaper is not necessarily better. Public Opin Q. 2007;71(4):635-48.

74. Atinc $M$, Yasmin $O$. Response rate and response error in marketing research - ProQuest [Internet]. 2012 [cited 2018 Aug 29]. Available from: https://search.proquest.com/openview/912c161dd4b8a19d6371453f4 20dd30a/1?pq-origsite $=$ gscholar\&cbl=18750\&diss $=y$

75. Walker BJ, Burdick RK. Advance correspondence and error in mail surveys. J Mark Res. 1977;14(3):379-82.

76. Snow RE, Prather JE, Hutcheson JD. Program evaluation using a follow-up telephone survey: the effects of a prior letter. Eval Rev. 1986;10(1):85-94.

77. Pitiyanuwat S, Phattharayuttawat S. Prenotification, Ink Color and Return Deadline: Effects on Response Rates and Sincerity of Responses [Internet]. 1991 [cited 2018 Aug 29]. Available from: https://eric.ed. gov/?id=ED334221

78. Nicolaas G, Smith P, Pickering K, Branson C. Increasing response rates in postal surveys while controlling costs: an experimental investigation. Soc Res Practice. 2015;1:3-15.

79. Link MW, Mokdad A. Advance letters as a means of improving respondent cooperation in random digit dial StudiesA multistate experiment. Public Opin Q. 2005;69(4):572-87.

80. Kulka R, Shirey S, Moore P, Woodbury N. A factorial experiment on the responses of professional nurses to a national mail survey [internet]. 1981. Available from: https://ww2.amstat.org/sections/srms/Proce edings/papers/1981_057.pdf

81. Kaplowitz MD, Hadlock TD, Levine R. A comparison of web and mail survey response rates. Public Opin Q. 2004;68(1):94-101.

82. Groves R, Snowden C. The effects of advanced letters on response rates in linked telephone surveys [internet]. 1987. Available from: http://ww2. amstat.org/sections/srms/Proceedings/papers/1987_113.pdf

83. Furse DH, Stewart DW, Rados DL. Effects of foot-in-the-door, cash incentives, and Followups on survey response. J Mark Res. 1981;18(4):473-8.

84. Chebat J-C, Cohen A. EBSCOhost | 9602160676 | Response speed in mail surveys: Beware of shortcuts. 1993 [cited 2018 Aug 29]. Available from: https://web.b.ebscohost.com/abstract?direct=true\&profile= 
ehost\&scope $=$ site\&authtype $=$ crawler\&jrn $=10408460 \& a s a=Y \& A N=$ 9602160676\&h=0pOj4bHcen6Nw9NApZ5UuSfC\%2bnykPtgMNh2\% 2bcmZtexko8G0KnRPsoycj5k9qLEJqkFFZxkAK7od4ajkWT\%2bhxvw\% $3 \mathrm{~d} \% 3 \mathrm{~d} \& \mathrm{crl}=\mathrm{c} \&$ resultNs=AdminWebAuth\&resultLocal=ErrCrINotAuth\& crlhashurl=login.aspx\%3fdirect\%3dtrue\%26profile\%3dehost\%26sco pe\%3dsite\%26authtype\%3dcrawler\%26jrn|\%3d10408460\%26asa\% 3dY\%26AN\%3d9602160676

85. Boser JA. Variations in Mail Survey Procedures: Comparison of Response Rates and Cost [Internet]. 1990. [cited 2018 Aug 29]. Available from: https://eric.ed.gov/?id=ED319803.

86. Bergsten JW, Weeks MF, Bryan FA. Effects of an advance telephone call in a personal interview survey. Public Opin Q. 1984;48(3):650-7.

87. Baulne J, Courtemanche R. Is there really any benefit in sending out introductory letters in Random Digit Dialling (RDD) surveys? ARCHIVED [Internet]. 2009 [cited 2018 Aug 29]. Available from: https:// www150.statcan.gc.ca/n1/pub/11-522-x/2008000/article/11001-eng. pdf.

88. Henri J-F, Thibodeau N. Follow-ups and mail survey response rates in management accounting research. 2012; Available from: https://www. researchgate.net/profile/Nicole_Thibodeau/publication/260638485 FOLLOW-UPS_AND_MAIL_SURVEY_RESPONSE_RATES_IN_MANAG EMENT_ACCOUNTING_RESEARCH/links/02e7e531e163cd3ace000000. pdf

89. Lalasz C, Doane M, Victoria S, Veronica D. Examining the Effect of Prenotification Postcards on Online Survey Response Rate in a University Graduate Sample. Surv Pract. 2014;7(3).

90. von der Lippe E, Schmich P, Lange C. Advance letters as a way of reducing non-response in a National Health Telephone Survey: differences between listed and unlisted numbers. Surv Res Methods. 2011;5(3):103-16.

91. Lusinchi D. Increasing response rates \& data quality of web surveys: pre-notification and questionnaire paging format. Far West Res. 2007.

92. McCallister LA, Otto B. Exploring the impact of E-mail and postcard Prenotification on response rates to a mail survey in an academic setting. J Appl Soc Sci. 2008;2(1):94-103.

93. Miner MH. Preliminary contact with a mailed follow-up survey: effect on rate of response of former mental health patients. Eval Rev. 1983;7(3):385-96.

94. Steeh C, Buskirk TD, Callegaro M. Using text messages in U.S. Mobile phone surveys. Field Methods. 2007;19(1):59-75.

95. Woodruff SI, Mayer JA, Clapp E. Effects of an introductory letter on response rates to a teen/parent telephone health survey. Eval Rev. 2006;30(6):817-23.

96. Traugott MW, Goldstein K. Evaluating dual frame samples and advance letters as a means of increasing response rates. InProceedings of the Survey Research Methods Section, American Statistical Association: 1993. pp. 1284-6.

97. Traugott M, Goldstein K. Evaluating dual frame samples and advance letters as a means of increasing response rates. Public Opin Q. 1987:51:522-39.

98. Traugott MW, Groves RM, Lepkowski JM. Using dual frame designs to reduce nonresponse in telephone surveys. Public Opin Q. 1987:51(4):522-39.

99. Brehm J. Stubbing our toes for a foot in the door? Prior contact, incentives and survey response. Int J Public Opin Res. 1994:6(1):45-63.

100. Camburn D, Inc AA, Lavrakas PJ, Battaglia MP. Using advance respondent letters in random-digit-dialing telephone surveys. In: Proceedings of the American Statistical Association Section on Survey Methods; 1995. p. 96997-4.

101. Dillman DA, Gallegos JG, Frey JH. Reducing refusal rates for telephone interviews. Public Opin Q. 1976;40(1):66-78.

102. Eyerman J, Link M, Mokdad A, Morton J. Assessing the Impact of Methodological Enhancements on Different Subpopulations in an Experiment on the Behavioral Risk Factor Surveillance System. Joint Statist Meet. 2003;1:1357-61.

103. Goldstein KM, Jennings MK. The effect of advance letters on cooperation in a list sample telephone survey. Public Opin Q. 2002;66(4):608-17.

104. Hembroff LA, Rusz D, Rafferty A, McGee H, Ehrlich N. The cost-effectiveness of alternative advance mailings in a telephone survey. Public Opin Q. 2005;69(2):232-45.
105. Iredell $H$, Shaw $T$, Howat P, James R, Granich J. Introductory postcards: do they increase response rate in a telephone survey of older persons? Health Educ Res. 2004:19(2):159-64.

106. Mickey R, Vacek P. Effects of survey mode and advance letters on contact and interview completion rates for population-based surveys of women. Proc Section Surv Res Methods Am Stat Assoc. 1999.

107. Smith W, Chey T, Jalaludin B, Salkeld G, Capon T. Increasing response rates in telephone surveys: a randomized trial. J Public Health (Oxf). 1995:17(1):33-8.

108. Singer E, Hoewyk V. JOHN, Maher MP. Experiments with incentives in telephone surveys. Public Opin Q. 2000;64(2):171-88.

109. Gerritsen M, Palmen M-J. The effect of prenotification techniques on refusal rate in telephone surveys: A real-life study in light of the compliance and elaboration likelihood theories. Doc Des. 2002:3(1):16-28.

110. Brick JM, Collins MA. A Response Rate Experiment for RDD Surveys. In: Proceedings of the American Statistical Association, Survey Research Methods Section; 1997. p. 1052-7.

111. Goulao B, Duncan A, Floate R, Clarkson J, Ramsay C. Three behavior change theory-informed randomized studies within a trial to improve response rates to trial postal questionnaires. J Clin Epidemiol. 2020;122:35-41.

112. Rodgers S, Sbizzera I, Cockayne S, Fairhurst C, Lamb SE, Vernon W, et al. A study update newsletter or Post-it ${ }^{\circledR}$ note did not increase postal questionnaire response rates in a falls prevention trial: an embedded randomised factorial trial. F1000Research. 2018;7.

113. Sakshaug JW, Vicari B, Couper MP. Paper, e-mail, or both? Effects of contact mode on participation in a web survey of establishments. Soc Sci Comput Rev. 2019;37(6):750-65.

114. von Allmen RS, Tinner C, Schmidli J, Tevaearai HT, Dick F. Randomized controlled comparison of cross-sectional survey approaches to optimize follow-up completeness in clinical studies. PLoS One. 2019;14(3):e0213822.

115. Griggs AK, Powell RJ, Keeney J, Waggy M, Harris KM, Halpern CT, et al. Research note: A prenotice greeting card's impact on response rates and response time. Longitudinal Life Course Stud. 2019;10(4):421-32.

116. Vogl S. Advance letters in a telephone survey on domestic violence: effect on unit nonresponse and reporting. Int J Public Opin Res. 2019:31(2):243-65.

117. Gooden T, Wright A, Swinn E, Sizmur S. Optimising response rates in a national postal survey evaluating community mental health care: four interventions trialled. J Ment Health. 2021;14:1-7.

118. Dijkers M. Introducing GRADE: a systematic approach to rating evidence in systematic reviews and to guideline development. KT Update. 2013;1(5):1-9.

119. Guyatt GH, Oxman AD, Kunz R, Brozek J, Alonso-Coello P, Rind D, et al. GRADE guidelines 6. Rating the quality of evidence-imprecision. J Clin Epidemiol. 2011;64(12):1283-93.

120. Lefebvre C, Manheimer E, Glanville J. Chapter 6: Searching for studies. In: Higgins JPT, Green S, editors. Cochrane Handbook for Systematic Reviews of Interventions. Version 5.1.0 [updated March 2011]: The Cochrane Collaboration; 2011. Available from www.cochrane-handb ook.org.

121. Eldridge S, Campbell M, Campbell M, Dahota A, Giraudeau B, Higgins J, Reeves B, Siegfried N. Revised Cochrane risk of bias tool for randomized trials (RoB 2.0): additional considerations for cluster-randomized trials.

122. Lacey RJ, Wilkie R, Wynne-Jones G, Jordan JL, Wersocki E, McBeth J. Evidence for strategies that improve recruitment and retention of adults aged 65 years and over in randomised trials and observational studies: a systematic review. Age Ageing. 2017;46(6):895-903.

123. van Gelder MMHJ, Vlenterie R, IntHout J, Engelen LLPG, Vrieling A, van de Belt TH. Most response-inducing strategies do not increase participation in observational studies: a systematic review and meta-analysis. J Clin Epidemiol. 2018;99:1-13.

124. Beebe TJ, Rey E, Ziegenfuss JY, Jenkins S, Lackore K, Talley NJ, et al. Shortening a survey and using alternative forms of prenotification: impact on response rate and quality. BMC Med Res Methodol. 2010;10(1):50

125. Bhutta MF, Hobson L, Lambie J, Scaman ESH, Burton MJ, Giele H, et al. Alternative recruitment strategies influence saliva sample return rates in community-based genetic association studies. Ann Hum Genet. 2013;77(3):244-50. 
126. Duncan A, Zajac I, Flight I, Stewart BJ, Wilson C, Turnbull D. Comparison of mailed invitation strategies to improve fecal occult blood test participation in men: protocol for a randomized controlled trial. Trials. 2013;14(1):239.

127. Dykema J, Stevenson J, Klein L, Kim Y, Day B. Effects of E-mailed versus mailed invitations and incentives on response rates, data quality, and costs in a web survey of university faculty. Soc Sci Comput Rev. 2013;31(3):359-70.

128. Edelman LS, Yang R, Guymon M, Olson LM. Survey methods and response rates among rural community dwelling older adults. Nurs Res. 2013;62(4):286.

129. Edwards L, Salisbury C, Horspool K, Foster A, Garner K, Montgomery AA. Increasing follow-up questionnaire response rates in a randomized controlled trial of telehealth for depression: three embedded controlled studies. Trials. 2016;17(1):107

130. Edwards P, Roberts I, Clarke M, DiGuiseppi C, Pratap S, Wentz R, et al. Methods to increase response rates to postal questionnaires. Cochrane Database Syst Rev. 2007;2:MR000008.

131. Gattellari M, Zwar N, Worthington JM. No difference demonstrated between faxed or mailed prenotification in promoting questionnaire response among family physicians: a randomized controlled trial. J Clin Epidemiol. 2012;65(5):544-52.

132. Grava-Gubins I, Scott S. Effects of various methodologic strategies: survey response rates among Canadian physicians and physicians-intraining. Can Fam Physician. 2008;54(10):1424-30.

133. Green $\mathrm{O}$, Ayalon L. Improving the cooperation rate of older adults and their caregivers in research surveys. GER. 2015;61(4):355-63.

134. Greenfield D, Moldovan $M$, Westbrook $M$, Jones $D$, Low $L$, Johnston $B$, et al. An empirical test of short notice surveys in two accreditation programmes. Int J Qual Health Care. 2012;24(1):65-71.

135. Hoisak JDP, Pawlicki T, Kim G-Y, Fletcher R, Moore KL. Improving linear accelerator service response with a real-time electronic event reporting system. J Appl Clin Med Phys. 2014;15(5):257-64.

136. Jacob RT, Jacob B. Prenotification, incentives, and survey modality: an experimental test of methods to increase survey response rates of school principals. J Res Educ Effectiveness. 2012;5(4):401-18.

137. Keusch F. How to increase response rates in list-based web survey samples. Soc Sci Comput Rev. 2012;30(3):380-8.

138. Koitsalu M, Eklund M, Adolfsson J, Grönberg $H$, Brandberg Y. Effects of pre-notification, invitation length, questionnaire length and reminder on participation rate: a quasi-randomised controlled trial. BMC Med Res Methodol. 2018;18(1):3.

139. Koopman L, Donselaar LCG, Rademakers JJ, Hendriks M. A prenotification letter increased initial response, whereas sender did not affect response rates. J Clin Epidemiol. 2013;66(3):340-8.

140. Leathem CS, Cupples ME, Byrne MC, O'Malley M, Houlihan A, Murphy $A W$, et al. Identifying strategies to maximise recruitment and retention of practices and patients in a multicentre randomised controlled trial of an intervention to optimise secondary prevention for coronary heart disease in primary care. BMC Med Res Methodol. 2009;9(1):40.

141. Libby G, Bray J, Champion J, Brownlee LA, Birrell J, Gorman DR, et al. Pre-notification increases uptake of colorectal Cancer screening in all demographic groups: A randomized controlled trial. J Med Screen. 2011;18(1):24-9.

142. Lynn P, Smith P, Turner R. Assessing the effects of an advance letter for a personal interview survey - ProQuest [Internet]. 1998 [cited 2018 Aug 29]. Available from: https://search.proquest.com/openview/c2ef659993 40318bed063df846910e7a/1?pq-origsite=gscholar\&cbl=45628.

143. Lynn P. Targeted appeals for participation in letters to panel survey members. Public Opin Q. 2016;80(3):771-82.

144. Man M-S, Rick J, Bower P, on behalf of the Healthlines Study Group, on behalf of the MRC-START Group. Improving recruitment to a study of telehealth management for long-term conditions in primary care: two embedded, randomised controlled trials of optimised patient information materials. Trials. 2015;16(1):309.

145. Martins $Y$, Lederman Rl, Lowenstein CL, Joffe S, Neville BA, Hastings BT, et al. Increasing response rates from physicians in oncology research: a structured literature review and data from a recent physician survey. $\mathrm{Br}$ J Cancer. 2012;106(6):1021-6.

146. Millar MM, Dillman DA. Improving response to web and mixed-mode surveys. Public Opin Q. 2011;75(2):249-69.

147. Mitchell V-W, Brown J. Research note: A cost-benefit analysis of letter prenotification and follow-up. J Mark Manag. 1997;13(8):853-66.

148. O'Carroll RE, Steele RJ, Libby G, Brownlee L, Chambers JA. Anticipated regret to increase uptake of colorectal cancer screening in Scotland (ARTICS): study protocol for a randomised controlled trial. BMC Public Health. 2013;13(1):849.

149. Senore C, Ederle A, DePretis G, Magnani C, Canuti D, Deandrea S, et al. Invitation strategies for colorectal cancer screening programmes: the impact of an advance notification letter. Prev Med. 2015;73:106-11.

150. Slater M, Kiran T. Measuring the patient experience in primary care: comparing e-mail and waiting room survey delivery in a family health team. Can Fam Physician. 2016;62(12):e740-8.

151. Todd AL, Porter M, Williamson JL, Patterson JA, Roberts CL. Pre-notification letter type and response rate to a postal survey among women who have recently given birth. BMC Med Res Methodol. 2015;15(1):104.

152. Treweek S, Mitchell E, Pitkethly M, Cook J, Kjeldstrøm M, Taskila T, et al. Strategies to improve recruitment to randomised controlled trials. Cochrane Database Syst Rev. 2010;1:MR000013.

153. Treweek S, Pitkethly M, Cook J, Fraser C, Mitchell E, Sullivan F, et al. Strategies to improve recruitment to randomised trials. Cochrane Database Syst Rev. 2018;22(2):MR000013.

154. Wagner J, Schroeder HM, Piskorowski A, Ursano RJ, Stein MB, Heeringa SG, et al. Timing the mode switch in a sequential mixed-mode survey: an experimental evaluation of the impact on final response rates, key estimates, and costs. Soc Sci Comput Rev. 2017;35(2):262-76.

155. Weiner MD, Puniello OT, Noland RB. Conducting efficient transit surveys of households surrounding transit-oriented developments. Transportation Res Record J Transportation Res Board. 2016;2594:44-50.

156. Westrick SC, Mount JK. Evaluating telephone follow-up of a mail survey of community pharmacies. Res Soc Adm Pharm. 2007;3(2):160-82.

157. Young T, Hopewell S. Methods for obtaining unpublished data. Cochrane Database Syst Rev. 2011;11:MR000027.

158. Social-research-practice-journal-issue-01-winter-2015.pdf [Internet]. [cited 2018 Aug 29]. Available from: http://the-sra.org.uk/wp-content/ uploads/social-research-practice-journal-issue-01-winter-2015.pdf\# page $=5$.

159. Murphy PM, Daley JM. Exploring the effects of postcard prenotification on industiral firms' response to mail surveys. J Mark Res Soc. 1991;33(4):335-41.

160. Scott FG. Mail questionnaires used in a study of older women. Sociol Soc Res. 1957:41:281-4.

161. Sack DI, Woodruff SI, McCabe CT, Galarneau MR, Han PP. Evaluation of three postal invitational strategies to increase survey response rates in a combat-injured US military population: findings from the Wounded Warrior Recovery Project. Mil Med. 2019;184(Supplement_1):521-8.

162. Harrison S, Henderson J, Alderdice F, Quigley MA. Methods to increase response rates to a population-based maternity survey: a comparison of two pilot studies. BMC Med Res Methodol. 2019;19(1):1-8.

163. Robbins MW, Grimm G, Stecher B, Opfer VD. A comparison of strategies for recruiting teachers into survey panels. SAGE Open. 2018;8(3):2158244018796412.

164. Schell C, Godinho A, Kushnir V, Cunningham JA. To send or not to send: weighing the costs and benefits of mailing an advance letter to participants before a telephone survey. BMC Res Notes. 2018;11(1):1-5.

165. Koitsalu M, Eklund M, Adolfsson J, Grönberg H, Brandberg Y. Effects of pre-notification, invitation length, questionnaire length and reminder on participation rate: a quasi-randomised controlled trial. BMC Med Res Methodol. 2018;18(1):1-5.

\section{Publisher's Note}

Springer Nature remains neutral with regard to jurisdictional claims in published maps and institutional affiliations. 